\title{
AN INVERSE THEOREM: WHEN THE MEASURE OF THE SUMSET IS THE SUM OF THE MEASURES IN A LOCALLY COMPACT ABELIAN GROUP
}

\author{
JOHN T. GRIESMER
}

\begin{abstract}
We classify the pairs of subsets $A, B$ of a locally compact abelian group $G$ satisfying $m_{*}(A+B)=m(A)+m(B)$, where $m$ is the Haar measure for $G$ and $m_{*}$ is inner Haar measure. This generalizes M. Kneser's classification of such pairs when $G$ is assumed to be connected. Recently, D. Grynkiewicz classified the pairs of sets $A, B$ satisfying $|A+B|=|A|+|B|$ in an abelian group, and our result is complementary to that classification. Our proofs combine arguments of Kneser and Grynkiewicz.
\end{abstract}

\section{INTRODUCTION}

1.1. Small sumsets in LCA groups. Given subsets $A$ and $B$ of an abelian group $G$, we consider their sumset $A+B:=\{a+b: a \in A, b \in B\}$. A general theme in additive combinatorics is that $A$ and $B$ must be highly structured when $A+B$ is not very large in comparison to $A$ and $B$; see [3], 17], or [21] for many instances of this theme. We consider the case where $G$ is a locally compact abelian (LCA) group, extending the investigations of [12]. Theorem 1 of [12] describes the pairs $(A, B)$ of Haar measurable subsets of an LCA group $G$ with Haar measure $m$ satisfying $m_{*}(A+B)<m(A)+m(B)$; such pairs are often called critical pairs. Here $m_{*}$ is the inner measure corresponding to $m$, so $m_{*}(S)=\sup \{m(E): E \subseteq S, E$ is compact $\}$. If $S \subseteq G$ and $t \in G$, we write $S+t$ for the set $\{s+t: s \in S\}$, and write $C \sim D$ if $m(C \triangle D)=0$.

Proposition 1.1 ([12, Theorem 1). Let $G$ be a locally compact abelian group with Haar measure $m$, and $A, B \subseteq G$ measurable sets with $m_{*}(A+B)<m(A)+m(B)$. Then the group $H:=\{t \in G: A+B+t \sim A+B\}$ is compact and open,

$$
A+B=A+B+H,
$$

and

$$
m(A+B)=m(A+H)+m(B+H)-m(H) .
$$

Proposition 1.1, especially equation (1.2), plays a crucial role in our results.

Since connected groups have no proper compact open subgroups, Proposition 1.1 implies $m_{*}(A+B) \geq \min \{m(A)+m(B), m(G)\}$ whenever $G$ is connected, generalizing results of Raikov [18, Macbeath [13], and Shields [20]. The following

Received by the editors April 14, 2012.

2010 Mathematics Subject Classification. Primary 11P70.

(C)2013 American Mathematical Society Reverts to public domain 28 years from publication 
theorem from 12 completely classifies the pairs of subsets of a compact, connected, abelian group satisfying $m_{*}(A+B)=m(A)+m(B)$, when $m(A)>0$ and $m(B)>0$. We call such a pair $(A, B)$ a sur-critical pair. Here $\mathbb{T}$ is the group $\mathbb{R} / \mathbb{Z}$; see $\$ 2.1 .1$ for our usage of the term "interval".

Proposition 1.2 ([12, Theorem 3$)$. Let $G$ be a compact connected abelian group with Haar measure $m$, and $A, B \subseteq G$ measurable sets satisfying $m(A)>0, m(B)>$ 0 , and $m_{*}(A+B)=m(A)+m(B)<1$. Then there is a continuous surjective homomorphism $\chi: G \rightarrow \mathbb{T}$, and there are intervals $I, J \subseteq \mathbb{T}$ such that $A \subseteq \chi^{-1}(I)$, $B \subseteq \chi^{-1}(J), m(A)=m\left(\chi^{-1}(I)\right)$, and $m(B)=m\left(\chi^{-1}(J)\right)$.

Kemperman [11 generalized part of Proposition 1.1 to the case where $G$ is not abelian, showing in particular that $m_{*}(A \cdot B) \geq m(A)+m(B)$ for subsets of a general locally compact connected group (here $A \cdot B$ denotes $\{a b: a \in A, b \in B\}$ ). Bilu [1] investigates the inequality $m(A+B)<m(A)+m(B)+\min \{m(A), m(B)\}$ when the ambient group is $\mathbb{T}^{d}$ for some $d$; the case $d=1$ is studied in [14].

1.2. New results. When $G$ is disconnected, the pairs $(A, B)$ of subsets of $G$ satisfying $m_{*}(A+B)=m(A)+m(B)$ are not completely described by Proposition 1.2. For example, take $G=(\mathbb{Z} / 5 \mathbb{Z}) \times \mathbb{T}$, and let $A=(\{0\} \times \mathbb{T}) \cup(\{1\} \times[0,1 / 2])$, $B=(\{0,1\} \times \mathbb{T}) \cup(\{2\} \times[0,1 / 4])$, so that $A+B=(\{0,1,2\} \times \mathbb{T}) \cup(\{1\} \times[0,3 / 4])$. Other examples are discussed in $\$ 4$. We classify the pairs $(A, B)$ of measurable subsets of a locally compact abelian group satisfying $m(A)>0, m(B)>0$, and $m_{*}(A+B)=m(A)+m(B)$, generalizing Proposition 1.2 to the case where $G$ may be disconnected. Our main result is Theorem 1.3, where $G$ is assumed to be compact. See $₫ 2$ for notation and terminology. In $\$ 7$ we discuss the case where $G$ is not compact.

Theorem 1.3. Let $G$ be a compact abelian group with Haar measure $m$, and let $A, B \subseteq G$ be measurable sets such that $m(A)>0, m(B)>0$, and $m_{*}(A+B)=$ $m(A)+m(B)$. Then at least one of the following is true:

(P) There is a compact open subgroup $K \leqslant G$ with $A+K \sim A$ and $B+K \sim B$.

(E) There are measurable sets $A^{\prime} \supseteq A$ and $B^{\prime} \supseteq B$ such that

$$
m\left(A^{\prime}\right)+m\left(B^{\prime}\right)>m(A)+m(B),
$$

and $m_{*}\left(A^{\prime}+B^{\prime}\right)=m_{*}(A+B)$.

(K) There is a compact open subgroup $K \leqslant G$, a continuous surjective homomorphism $\chi: K \rightarrow \mathbb{T}$, intervals $I, J \subseteq \mathbb{T}$, and elements $a, b \in G$ such that $A \subseteq a+\chi^{-1}(I), B \subseteq b+\chi^{-1}(J), m(A)=m\left(\chi^{-1}(I)\right)$, and $m(B)=m\left(\chi^{-1}(J)\right)$.

(QP) There is a compact open subgroup $K \leqslant G$ and partitions $A=A_{1} \cup A_{0}$, $B=B_{1} \cup B_{0}$ such that $A_{0} \neq \varnothing, B_{0} \neq \varnothing$, at least one of $A_{1} \neq \varnothing, B_{1} \neq \varnothing$, and

(QP.1) $A_{1}+K \sim A_{1}, B_{1}+K \sim B_{1}$, while $A_{0}$ and $B_{0}$ are each contained in a coset of $K$ and $\left(A_{1}+K\right) \cap A_{0}=\left(B_{1}+K\right) \cap B_{0}=\varnothing$;

(QP.2) $A_{0}+B_{0}+K$ is a unique expression element of $A+B+K$ in $G / K$;

(QP.3) $m_{*}\left(A_{0}+B_{0}\right)=m\left(A_{0}\right)+m\left(B_{0}\right)$.

Remarks. (i) Corollary 3.1 provides more detail in conclusion (QP); conclusion (E) is examined in 3.3 . Corollary 3.3 is a convenient rephrasing of Theorem 1.3. 
(ii) We do not provide explicit constructions of all pairs satisfying conclusion (QP), so the statement of Theorem 1.3 is not a complete characterization of the sur-critical pairs for a compact abelian group.

(iii) The labels (P), (E), (K), and (QP) stand for "periodic", "extendible", "Kneser", and "quasi-periodic", respectively. See \$2.2 for elaboration.

(iv) Theorem 1.3 was developed partly to help answer Question 4.1 of [9].

Theorem 1.3 is proved in 96.2 . A sequence of lemmas in 95 reduces the proof to a special case. We then apply the $e$-transform (\$5.6) as in the proof of Proposition 1.2 from [12. Lemma 5.20 handles difficulties related to applying the $e$-transform in a disconnected group; its statement and proof are inspired by arguments from [4].

Some easy consequences of Theorem 1.3 are discussed in 33 Examples are given in 4 showing that each conclusion in Theorem 1.3 may occur, and none can be omitted. 43 and 84 are technically irrelevant to the proof of Theorem 1.3 .

1.3. When one of $m(A)=0$ or $m(B)=0$. When $m(B)=0,0<m(A)<\infty$, and $B$ contains the identity element of $G$, Theorem 4 of [12] says that $m_{*}(A+B)=$ $m(A)+m(B)$ if and only if the closed subgroup $G^{\prime} \leqslant G$ generated by $B$ is compact, and $A$ can be partitioned as $A=C \cup D$, where $m(C)=0, m_{*}(B+C)=0$ and $m\left(G^{\prime}+D\right)=m(D)$. We have nothing to say regarding the case where $m(A)=$ $m(B)=0$.

1.4. Context. The conclusion of Theorem 1.3 is trivial for discrete groups $G$, where the trivial subgroup $\{0\}$ is compact and open, so conclusion $(\mathrm{P})$ holds with $K=\{0\}$. The article 44 classifies the sur-critical pairs for discrete groups, and therefore provides a classification of the pairs $(A, B)$ satisfying conclusion $(\mathrm{P})$ in Theorem 1.3 (see 4.1). We summarize the development of some related results.

Inverse theorems in additive combinatorics deduce properties of subsets $A, B$ of an abelian group from a hypothesis on the sumset $A+B$, while direct theorems deduce properties of $A+B$ from hypotheses on $A$ and $B$. One of the earliest direct theorems is the Cauchy-Davenport inequality, which states that $|A+B| \geq$ $\min \{|A|+|B|-1, p\}$ whenever $A, B \subseteq \mathbb{Z} / p \mathbb{Z}$ for some prime $p$; here $|S|$ denotes the cardinality of the set $S$. The corresponding inverse theorem, due to Vosper [22,23, classifies the pairs $(A, B)$ of subsets of $G=\mathbb{Z} / p \mathbb{Z}$ satisfying $|A+B|=|A|+|B|-1$, when $|A|+|B|<p$ : the equation holds if and only if $A$ and $B$ are arithmetic progressions with the the same common difference, or one of $|A|=1,|B|=1$. When $|A|+|B|=p$, an elementary analysis shows that the equation $|A+B|=|A|+|B|-1$ is satisfied exactly when $B$ is a translate of $-(G \backslash A)$, and when $|A|+|B|=p+1$, the equation $|A+B|=|A|+|B|-1$ is always satisfied if $A$ and $B$ are nonempty.

Generalizing Vosper's theorem and strengthening Proposition 1.1, Kemperman [10] classified the pairs of finite subsets $A, B$ of an arbitrary abelian group satisfying $|A+B|<|A|+|B|$; see [3] for exposition. The articles [5, 6] classify pairs $(A, B)$ satisfying $|A+B|=|A|+|B|$ in certain ambient abelian groups. More recently, Grynkiewicz [4] classified the pairs $(A, B)$ of finite subsets of an arbitrary abelian group satisfying $|A+B|=|A|+|B|$. That classification is somewhat intricate, so we do not reproduce it here. Concatenating Theorem 1.3 with the results of [10] and 4 yields a very precise description of pairs $(A, B)$ of subsets of a compact abelian group satisfying $m_{*}(A+B)=m(A)+m(B)$, but we will not state this description explicitly. 


\section{Terminology, notation, And BACKGround}

We assume knowledge of the theory of locally compact abelian groups. The books [8] and [19] together provide nearly sufficient background, as does [12] and its bibliography. We need Theorem A of [15], which is proved in [16].

Some of our terminology and notation is taken from [4] and [12]; when $G$ is discrete, some of our definitions coincide with those from [4].

2.1. General conventions. Throughout, $G$ will denote a locally compact abelian group and $m_{G}$ will be its Haar measure. We make the standard assumption that $G$ satisfies the $T_{0}$ separation axiom. When there is no chance of confusion, we write $m$ for $m_{G}$, and $m_{*}$ for the corresponding inner measure. The term measurable in reference to a subset of $G$ will always mean "lies in the completion of the Borel $\sigma$-algebra with respect to $m$ ". We will exploit the inner regularity of the Haar measure for compact groups: $m(C)=\sup \{m(E): E \subseteq C, E$ is compact $\}$ when $C$ is measurable and $G$ is compact. The Haar measure will always be normalized for compact groups, so $m_{G}(G)=1$ for such $G$.

If $S \subseteq G, S^{c}$ will denote the set theoretic complement $G \backslash S$. The symbol $-S$ denotes $\{-s: s \in S\}$, while $A-B$ denotes the difference set $\{a-b: a \in A, b \in B\}$.

The identity element of $G$ will be written as $0_{G}$, or simply 0 if there is no ambiguity.

2.1.1. Intervals. The symbol $\mathbb{T}$ will denote the group $\mathbb{R} / \mathbb{Z}$ with its usual topology, and "an interval in $\mathbb{T}$ " means a set of the form $[x, y]+\mathbb{Z}$, where $x \leq y<x+1$. We omit the term "closed", as we will never refer to nonclosed intervals. We frequently exploit the following property of intervals: if $I, J \subseteq \mathbb{T}$ are intervals with $m_{\mathbb{T}}(I)+m_{\mathbb{T}}(J)<1$, and $x \notin I$, then $m_{\mathbb{T}}((x+J) \backslash(I+J))>0$. Consequently, if $\chi: G \rightarrow \mathbb{T}$ is a continuous surjective homomorphism and $t \notin \chi^{-1}(I)$, then $m_{G}((t+\tilde{J}) \backslash(\tilde{I}+\tilde{J}))>0$, where $\tilde{I}=\chi^{-1}(I)$ and $\tilde{J}=\chi^{-1}(J)$.

2.1.2. Subgroups and quotients. If $K \leqslant G$ is a closed subgroup, the quotient $G / K$ with the quotient topology is a locally compact abelian group. We may identify subsets of $G$ of the form $A+K$ with subsets of $G / K$, and conversely subsets of $G / K$ may be identified with subsets of $G$.

A $K$-coset decomposition of a set $A \subseteq G$ is the collection of sets $A_{i}=A \cap K_{i}$, where $K_{i}$ ranges over the cosets of $K$ having nonempty intersection with $A$.

We use without comment the following well-known facts:

- If $K \leqslant G$ is a measurable subgroup of a compact group, then $m(K)>0$ if and only if $K$ is open, if and only if $K$ has finite index in $G$. The index of $K$ in $G$ is $1 / m(K)$.

- If $K \leqslant G$ is an open subgroup, the group $G / K$ is discrete.

2.1.3. Disintegration of Haar measure. This subsection regards technicalities occurring only in \$5.4. For simplicity we assume $G$ is compact. Given a closed subgroup $K \leqslant G$, we consider the Haar measure $m_{K}$ as a measure on $G$. If $A \subseteq G$ and $x \in G$, we consider $m_{K}(A-x)$, which may be regarded as the $m_{K}$-measure of $A$ in the coset $x+K$ of $K$. Although the function $g(x):=m_{K}(A-x)$ depends only on the coset $x+K$, and may therefore be regarded as a function whose domain is (a subset of) $G / K$, we prefer to regard $g$ as a function whose domain is (a subset of) $G$. The measure $m_{G}(A)$ can be recovered in a natural way from the measures $m_{K}(A-x)$; in other words, Haar measure can be disintegrated over the cosets of 
a closed subgroup. This is the content of the following proposition; cf. $\S 2$ of 12 . It may be obtained by specializing Theorem 3.4.6 of [19] to the case where $G$ is compact and abelian.

Proposition 2.1. Let $K \leqslant G$ be a closed subgroup of $G$ with Haar measure $m_{K}$, and let $f \in L^{1}(m)$ be a real-valued function. Then the function $\tilde{f}: G \rightarrow \mathbb{R}$ given by $\tilde{f}(x)=\int f(x+t) d m_{K}(t)$ is defined for $m$-almost every $x, \tilde{f}$ is $m_{G}$-measurable, and $\int \tilde{f} d m=\int f d m$. In particular, if $A \subseteq G$ is measurable, then the function $x \mapsto m_{K}(A-x)$ is measurable, and $\int m_{K}(A-x) d m(x)=m(A)$.

2.1.4. Unique expression elements. We say $c \in A+B$ is a unique expression element of $A+B$ if $c=a_{0}+b_{0}$ for some $a_{0} \in A, b_{0} \in B$, and $a+b=c$ implies $a=a_{0}$ and $b=b_{0}$ when $a \in A$ and $b \in B$. Unique expression elements play an important role in [10], the classification of pairs $(A, B)$ satisfying $|A+B|=|A|+|B|-1$; Corollary 3.1 connects that classification to conclusion (QP) of Theorem 1.3. If $K \leqslant G$ is a subgroup, the phrase " $C+K$ is a unique expression element of $A+B+K$ in $G / K$ " means that $C+K=a_{0}+b_{0}+K$ for some $a_{0} \in A, b_{0} \in B$, and whenever $a \in A$, $b \in B$, and $a+b+K=C+K$, then $a+K=a_{0}+K$ and $b+K=b_{0}+K$.

2.1.5. Critical and sur-critical pairs. If $A, B \subseteq G$ are measurable sets satisfying $m_{*}(A+B)=m(A)+m(B)$, we call $(A, B)$ a sur-critical pair for $G$. We call a pair $(A, B)$ satisfying $m_{*}(A+B)<m(A)+m(B)$ a critical pair for $G$.

2.1.6. Similarity and the essential stabilizer. If $C, D \subseteq G$, we write $C \sim D$ to mean $m(C \triangle D)=0$, and we write $C \subset_{m} D$ to mean $m(C \backslash D)=0$.

When $C$ is measurable and $m(C)<\infty$, the group $H(C):=\{t \in G: C+t \sim C\}$ is a compact subgroup of $G([12$, Lemma 4); we may refer to $H(C)$ as the essential stabilizer of $C$. The group $H(A+B)$ plays an important role in [12] and in the proof of Theorem 1.3. Proposition 1.1 says that if $m_{*}(A+B)<m(A)+m(B)$, then $H(A+B)$ is compact and open, and $A+B+H(A+B)=A+B$. The equation $C=$ $C+H(C)$ may fail in general, and more importantly, the similarity $C \sim C+H(C)$ may fail. For example, take $G=(\mathbb{Z} / 5 \mathbb{Z}) \times \mathbb{T}$, and let $C=(\{0\} \times \mathbb{T}) \cup\{(1,0)\}$. Then $H(C)=\{0\} \times \mathbb{T}$, so $m(C+H(C))=2 / 5$, while $m(C)=1 / 5$.

\subsection{Special sets and special pairs.}

2.2.1. Periodicity. If $C \sim C+K$ for some compact open subgroup $K \leqslant G$, we call $C$ periodic with period $K$. Otherwise, we call $C$ aperiodic. Note that when $G$ is not discrete, the assertion $H(C)=\{0\}$ implies $C$ is aperiodic, but an aperiodic $C$ may have $m(H(C))>0$. We will exploit the following relation between the essential stabilizer and periodicity. This observation is a consequence of Lemma 5.11, although it may be obtained by more elementary means.

Observation. If $m(H(C))>0$, then for all cosets $H_{i}$ of $H(C)$, either $m\left(C \cap H_{i}\right)=$ 0 or $m\left(C \cap H_{i}\right)=m\left(H_{i}\right)$. Consequently, if $m(H(C))>0$, there is a measurable subset $C^{\prime} \subseteq C$ such that $C \sim C^{\prime} \sim C^{\prime}+H(C)$.

Remark. When $G$ is discrete, every subset of $G$ is periodic according to our definition. This differs from the terminology of [4, where a periodic set $S$ must satisfy $S+t \sim S$ for some $t \neq 0$. 
2.2.2. Extendibility and nonextendibility. Let $A$ and $B$ be measurable subsets of a compact abelian group $G$. We say that $A$ is extendible with respect to $B$ if there is a measurable set $A^{\prime} \supseteq A$ with $m\left(A^{\prime}\right)>m(A)$ and $m_{*}\left(A^{\prime}+B\right)=m_{*}(A+B)$. We say that the pair $(A, B)$ is extendible if $A$ is extendible with respect to $B$ or $B$ is extendible with respect to $A$. Otherwise, we say that $(A, B)$ is nonextendible. The nonextendibility of a pair $(A, B)$ may be expressed as follows: if $A^{\prime} \supseteq A$ and $B^{\prime} \supseteq B$ are measurable and $m_{*}\left(A^{\prime}+B^{\prime}\right)=m_{*}(A+B)$, then $A^{\prime} \sim A$ and $B^{\prime} \sim B$.

When $m_{*}(A+B)=m(A)+m(B)$ and $(A, B)$ is extendible, Proposition 1.1 implies $H(A+B)$ is compact and open, and $A+B \sim A+B+H(A+B)$. Consequently, $A+B$ is measurable when $(A, B)$ is extendible. The following example lemma illustrates how nonextendibility will be exploited in subsequent proofs.

Lemma 2.2. If $K \leqslant G$ is a compact open subgroup, $A+B \sim A+B+K$, and $(A, B)$ is nonextendible, then $A+K \sim A$.

Proof. The similarity $A+B \sim A+B+K$ can be rewritten as $(A+K)+B \sim A+B$. Since $A+K \supseteq A$ and $(A, B)$ is nonextendible, we have $A+K \sim A$.

In subsequent proofs, such as those of Lemmas 5.5, 5.6, 5.17, and 5.20, we will omit the above reasoning.

2.2.3. Complementary pairs. If $G$ is compact and $m(A+B)=m(A)+m(B)=1$, call $(A, B)$ a complementary pair. When $G$ is infinite, it is easy to construct such pairs $(A, B)$ with $A+B \neq G$ : let $A \subseteq G$ be any measurable set meeting every coset of every finite index subgroup of $G$ with $0<m(A)<1$, and let $B \subseteq G$ have $m(B)=1-m(A)$. Then $(A, B)$ is a complementary pair by Proposition 1.1. In particular, if $G$ is connected and $A$ is any measurable subset of $G$, then $\left(A,-A^{c}\right)$ is a complementary pair.

Complementary pairs $(A, B)$ with $m(A)>0$ and $m(B)>0$ satisfying $A+B \neq G$ can be described as follows. If $(A, B)$ is a complementary pair, and $A+B \neq G$, then $A \cap(t-B)=\varnothing$ for some $t \in G$, so $t-B \sim A^{c}$. If $s \notin H(t-B)\left(=H\left(A^{c}\right)=H(A)\right)$, then $m(A \cap(t+s-B))>0$, so $t+s \in A+B$. It follows that $A+B$ contains a translate of $G \backslash H(A)$.

If $K \leqslant G$ is a compact open subgroup, $A$ and $B$ are each contained in a coset of $K$, and $m(A+B)=m(A)+m(B)=m(K)$, we say that $(A, B)$ is complementary with respect to $K$. Such pairs form a subclass of the extendible pairs.

2.2.4. Reducibility. If there are measurable subsets $A^{\prime} \subseteq A$ and $B^{\prime} \subseteq B$ such that $m\left(A^{\prime}\right)=m(A), m\left(B^{\prime}\right)=m(B)$, and $m_{*}\left(A^{\prime}+B^{\prime}\right)<m_{*}(A+B)$, we say that $(A, B)$ is reducible. Otherwise, we say $(A, B)$ is irreducible.

2.2.5. Quasi-periodicity. Let $K \leqslant G$ be a compact open subgroup. A set $A \subseteq G$ is called quasi-periodic with respect to $K$ if $A$ can be partitioned into two nonempty sets $A_{1}$ and $A_{0}$ such that $\left(A_{1}+K\right) \cap\left(A_{0}+K\right)=\varnothing, A_{1} \sim A_{1}+K$, and $A_{0}$ is contained in a coset of $K$. We call $A_{1} \cup A_{0}$ a quasi-periodic decomposition of $A$, and we say $K$ is a quasi-period of $A$. Note that $A$ may have more than one quasi-period.

Call a pair $(A, B)$ of subsets of $G$ quasi-periodic with respect to $K$ if one of $A$ or $B$ is quasi-periodic with respect to $K$ and the other is either contained in a coset of $K$ or is quasi-periodic with respect to $K$. This means that $A=A_{1} \cup A_{0}$, $B=B_{1} \cup B_{0}, A_{1} \sim A_{1}+K, B_{1} \sim B_{1}+K, A_{0}$ and $B_{0}$ are nonempty and contained in cosets of $K,\left(A_{1}+K\right) \cap A_{0}=\left(B_{1}+K\right) \cap B_{0}=\varnothing$, and at least one of $A_{1}$ and $B_{1}$ 
is nonempty. We say $A_{1} \cup A_{0}, B_{1} \cup B_{0}$ is a quasi-periodic decomposition of $(A, B)$, and $K$ is a quasi-period of $(A, B)$. Note that if $(A, B)$ is quasi-periodic with respect to $K$, then $A+B$ is quasi-periodic with respect to $K$.

Remark. Our insistence that $A_{1}$ be nonempty for $A$ to be quasi-periodic differs from the convention of [4]; the analogous definition in [4] allows $A_{1}=\varnothing$.

2.2.6. Repeated decompositions. If $A_{1} \cup A_{0}$ is a quasi-periodic decomposition of $A$ with quasi-period $K_{0}$ and $A_{0}=C_{1} \cup C_{0}$ is a quasi-periodic decomposition of $A_{0}$ with quasi-period $K_{1} \leqslant K_{0}$, then setting $A_{0}^{\prime}=C_{0}$ and $A_{1}^{\prime}=A \backslash C_{0}$, the partition $A=A_{1}^{\prime} \cup A_{0}^{\prime}$ is a quasi-periodic decomposition of $A$ with respect to $K_{1}$. Consequently, if $(A, B)$ satisfies (QP) of Theorem 1.3 with $A=A_{1} \cup A_{0}$, $B=B_{1} \cup B_{0}$, and subgroup $K=K_{1} \leqslant G$, and $\left(A_{0}, B_{0}\right)$ also satisfies (QP) with subgroup $K=K_{2} \leqslant G$, then $(A, B)$ satisfies (QP) with subgroup $K=K_{2} \leqslant G$.

\section{Consequences of Theorem 1.3}

3.1. Conclusion (QP) and critical pairs for finite groups. Sur-critical pairs (2.1.5) satisfying (QP) of Theorem 1.3 can be further described in terms of certain critical pairs for finite groups, as the following corollary shows.

Corollary 3.1. With the hypotheses and notation of Theorem 1.3 , if $(A, B)$ satisfies conclusion $(\mathrm{QP})$, then with $K$ being the subgroup of that conclusion,

(QP.4) $m(A+B+K)=m(A+K)+m(B+K)-m(K)$.

Viewing $A^{\prime}=A+K$ and $B^{\prime}=B+K$ as subsets of the discrete group $G / K$, (QP.4) means $\left|A^{\prime}+B^{\prime}\right|=\left|A^{\prime}\right|+\left|B^{\prime}\right|-1$, while (QP.2) says that $A^{\prime}+B^{\prime}$ has a unique expression element. The results of [10] may be used to classify such pairs $\left(A^{\prime}, B^{\prime}\right)$; see $\S 2$ of [3] for exposition.

Proof. Let $A=A_{1} \cup A_{0}, B=B_{1} \cup B_{0}$ constitute a pair satisfying (QP) and $m_{*}(A+B)=m(A)+m(B)$, and let $K$ be the corresponding subgroup. Note that

$$
m(A+K)=m\left(A_{1}\right)+m(K)=m(A)-m\left(A_{0}\right)+m(K),
$$

and a similar identity holds for $m(B+K)$. Since $A_{0}+B_{0}+K$ is a unique expression element of $A+B+K$ in $G / K$, we have

$$
\begin{aligned}
m(A+B+K) & =m_{*}(A+B)-m_{*}\left(A_{0}+B_{0}\right)+m(K) \\
& =m(A)-m\left(A_{0}\right)+m(B)-m\left(B_{0}\right)+m(K) \\
& =[m(A+K)-m(K)]+[m(B+K)-m(K)]+m(K) \\
& =m(A+K)+m(B+K)-m(K),
\end{aligned}
$$

where the second line uses the hypothesis and (QP.3) of Theorem 1.3, and the third uses (3.1).

As a counterpart to Corollary 3.1, the following procedure produces sur-critical pairs satisfying (QP) from critical pairs for finite groups.

Let $K \leqslant G$ be a compact open subgroup so that the quotient $F=G / K$ is discrete; write $\phi: G \rightarrow F$ for the quotient map. Let $\left(A^{\prime}, B^{\prime}\right)$ be a critical pair for $F$ such that $A^{\prime}+B^{\prime}$ has a unique expression element and $\left|A^{\prime}+B^{\prime}\right|=\left|A^{\prime}\right|+\left|B^{\prime}\right|-1$. Pick $a^{\prime} \in A^{\prime}, b^{\prime} \in B^{\prime}$ so that $a^{\prime}+b^{\prime}$ is a unique expression element of $A^{\prime}+B^{\prime}$. Define $A_{1}^{\prime}:=A^{\prime} \backslash\left\{a^{\prime}\right\}$ and $B_{1}^{\prime}:=B^{\prime} \backslash\left\{b^{\prime}\right\}$. Set $A_{1}=\phi^{-1}\left(A_{1}^{\prime}\right), B_{1}=\phi^{-1}\left(B_{1}^{\prime}\right)$, and choose 
$C \subseteq K$ and $D \subseteq K$ such that $m_{*}(C+D)=m(C)+m(D)$. Let $A_{0}=\phi^{-1}\left(a^{\prime}\right)+C$, $B_{0}=\phi^{-1}\left(b^{\prime}\right)+D$, and let $A=A_{1} \cup A_{0}, B=B_{1} \cup B_{0}$. Then

$$
m(A)=m(K)\left(\left|A^{\prime}\right|-1\right)+m(C), m(B)=m(K)\left(\left|B^{\prime}\right|-1\right)+m(D),
$$

and

$$
\begin{aligned}
m_{*}(A+B) & =m(K)\left(\left|A^{\prime}+B^{\prime}\right|-1\right)+m_{*}(C+D) \\
& =m(K)\left(\left|A^{\prime}\right|+\left|B^{\prime}\right|-2\right)+m(C)+m(D) \\
& =m(A)+m(B) .
\end{aligned}
$$

\subsection{Measurability and topology of $A, B$, and $A+B$.}

3.2.1. Essential regularity. For $S \subseteq G$, let $\bar{S}$ denote the topological closure of the set $S$, and let int $S$ denote the interior of $S$. Call a set $S \subseteq G$ essentially regular if $m_{*}(S)=m(\bar{S})=m($ int $\bar{S})$, and call a pair $(A, B)$ essentially regular if $A, B$, and $A+B$ are essentially regular. Proposition 1.2 implies $(A, B)$ is essentially regular when $G$ is a compact connected group and $A, B \subseteq G$ have $m(A)>0, m(B)>0$, and $m(A+B)=m(A)+m(B)$. Corollary 3.4 below shows that restricting the measures of $A$ and $B$ in Theorem 1.3 can guarantee that $(A, B)$ is essentially regular. For this we need the following lemma.

Lemma 3.2. If for all $\varepsilon>0,(A, B)$ has a quasi-periodic decomposition (\$2.2.5) with respect to a compact open subgroup $K$ having $0<m(K)<\varepsilon$, then $(A, B)$ is essentially regular.

Proof. Let $\varepsilon>0$. For a given quasi-period $K$ of $(A, B)$ having $0<m(K)<\varepsilon$, write $A=A_{1} \cup A_{0}$, where $A_{1} \sim A_{1}+K$ and $A_{0}$ is contained in a coset of $K$. Then $m(\bar{A}) \leq m(A+K) \leq m(A)+\varepsilon$, while $m(\operatorname{int} \bar{A}) \geq m(A+K)-m(K) \geq m(A)-\varepsilon$. Letting $\varepsilon \rightarrow 0$, we get that $m(A)=m(\bar{A})=m(\operatorname{int} \bar{A})$. The same argument applied to $B$ and $A+B$ shows that $B$ and $A+B$ are essentially regular.

3.2.2. Refinement of Theorem 1.3. In conclusion (QP) of Theorem 1.3, possibly $m\left(A_{0}\right)=0, m\left(B_{0}\right)=0$, or both. When $m\left(A_{0}\right)>0$ and $m\left(B_{0}\right)>0$, (QP.3) guarantees that Theorem 1.3 applies to the pair $\left(A_{0}, B_{0}\right)$. This observation yields the following corollary.

Corollary 3.3. With the hypotheses and notation of Theorem 1.3, at least one of the following is true:

(I) One of $(\mathrm{P}),(\mathrm{E})$, or $(\mathrm{K})$ holds.

(II) Conclusion (QP) holds and $\left(A_{0}, B_{0}\right)$ satisfies $(\mathrm{K})$.

(III) For all $\varepsilon>0$ there is a compact open subgroup $H \leqslant G$ having $m(H)<\varepsilon$ and $(A, B)$ satisfies $(\mathrm{QP})$ with $K=H$.

(IV) $(A, B)$ satisfies (QP) with at least one of $m\left(A_{0}\right)=0$ or $m\left(B_{0}\right)=0$.

Proof. If $(A, B)$ satisfies $(\mathrm{P}),(\mathrm{E})$, or $(\mathrm{K})$, we have (I). If not, inductively form a sequence of pairs $\left(A^{(n)}, B^{(n)}\right)$ and subgroups $K^{(n)} \leqslant G$ as follows: let $\left(A^{(0)}, B^{(0)}\right)=$ $(A, B)$, and let $K^{(0)}=G$. Suppose $\left(A^{(j)}, B^{(j)}\right)$ and $K^{(j)}$ are defined for $j=0, \ldots, n$ and for each $j=1, \ldots, n$,

(1) $A=A_{1}^{\prime} \cup A^{(j)}, B=B_{1}^{\prime} \cup B^{(j)}$ is a quasi-periodic decomposition of $(A, B)$ with respect to $K^{(j)}$ satisfying (QP) of Theorem 1.3, so that

(2) $A^{(j)}$ and $B^{(j)}$ are each contained in cosets of $K^{(j)}$, 
(3) $m_{*}\left(A^{(j)}+B^{(j)}\right)=m\left(A^{(j)}\right)+m\left(B^{(j)}\right)$, and

(4) $K^{(j)}$ is a proper subgroup of $K^{(j-1)}$.

We will construct $\left(A^{(n+1)}, B^{(n+1)}\right)$ and $K^{(n+1)} \leqslant K^{(n)}$ satisfying (1)-(4), or show that one of (I), (II), or (IV) holds.

If one or both of $m\left(A^{(n)}\right)=0$ or $m\left(B^{(n)}\right)=0$, we have conclusion (IV). Otherwise, apply Theorem 1.3 to $\left(A^{(n)}, B^{(n)}\right)$. If $\left(A^{(n)}, B^{(n)}\right)$ satisfies $(\mathrm{P})$ or $(\mathrm{E})$, then so does $(A, B)$, and we have (I). If $\left(A^{(n)}, B^{(n)}\right)$ satisfies (K), we have (II). Otherwise, $\left(A^{(n)}, B^{(n)}\right)$ satisfies $(\mathrm{QP})$, so take $K^{(n+1)}$ to be corresponding subgroup $K$, and let $A^{(n)}=A_{1}^{(n)} \cup A_{0}^{(n)}, B^{(n)}=B_{1}^{(n)} \cup B_{0}^{(n)}$ be the corresponding decompositions. Observe that $K^{(n+1)}$ must be a proper subgroup of $K^{(n)}$, so that $m\left(K^{(n+1)}\right) \leq \frac{1}{2} m\left(K^{(n)}\right)$. Now take $A^{(n+1)}=A_{0}^{(n)}$ and $B^{(n+1)}=B_{0}^{(n)}$, so that $\left(A^{(n+1)}, B^{(n+1)}\right)$ satisfies (1)-(4) with $j=n+1$ (cf. \$2.2.6).

If the above construction terminates, we conclude (I), (II), or (IV). Otherwise, we have for each $n$ quasi-periodic decompositions with respect to $K^{(n)}$, and we conclude (III).

Corollary 3.4. Suppose $(A, B)$ satisfies the hypotheses of Theorem 1.3 .

(a) If $A+B$ is not measurable, then $(A, B)$ satisfies (QP) with at least one of $m\left(A_{0}\right)=0$ or $m\left(B_{0}\right)=0$.

(b) If $(A, B)$ is not essentially regular, then $(A, B)$ satisfies conclusion (E) of Theorem 1.3, or $(A, B)$ satisfies conclusion (QP) of Theorem 1.3 with one or both of $m\left(A_{0}\right)=0$ or $m\left(B_{0}\right)=0$.

(c) If $m(A), m(B)$, and $m(A+B)$ are all irrational numbers, then $(A, B)$ is essentially regular.

Proof. (a) In conclusions (I) and (II) of Corollary 3.3, it is routine to check that $A+B$ is measurable. If (III) holds in Corollary 3.3. Lemma 3.2 implies $A+B$ is measurable.

(b) If $(\mathrm{P})$ or $(\mathrm{K})$ holds, then $(A, B)$ is essentially regular. If (II) or (III) of Corollary 3.3 holds, then $(A, B)$ is essentially regular. The only remaining alternatives are (E) of Theorem 1.3 or (IV) of Corollary 3.3

(c) If $m(A+B)$ is irrational, then neither conclusion (P) nor (E) can hold in Theorem 1.3. If $m(A)$ and $m(B)$ are irrational, then (QP) cannot hold with one of $m\left(A_{0}\right)=0$ or $m\left(B_{0}\right)=0$. Now $(A, B)$ is essentially regular, by part (b) of the present corollary.

3.3. Further description of extendible pairs. If $(A, B)$ is a sur-critical pair satisfying conclusion (E) of Theorem 1.3. Proposition 1.1 implies the group $H:=$ $H(A+B)$ is compact and open and $A+B \sim A+B+H$. Furthermore, exactly one of the following holds:

(E.1) $A+B=A+B+H$.

(E.2) $(A, B)$ is complementary with respect to $H$ and $A+B \neq A+B+H$, or $(A, B)$ satisfies conclusion (QP) of Theorem 1.3. with $K=H, m\left(A_{0}\right)+m\left(B_{0}\right)=$ $m(H)$, and $A_{0}+B_{0} \neq A_{0}+B_{0}+H$.

When $m\left(A_{0}\right)>0$ and $m\left(B_{0}\right)>0$ in (E.2), the pair $\left(A_{0}, B_{0}\right)$ is complementary with respect to the subgroup $H$; see $\$ 2.2 .3$ for further description.

To obtain this classification, fix $A^{\prime} \supseteq A$ and $B^{\prime} \supseteq B$ such that $m\left(A^{\prime}\right)+m\left(B^{\prime}\right)>$ $m(A)+m(B)$ and $m\left(A^{\prime}+B^{\prime}\right)=m(A+B)$, and write $H$ for $H\left(A^{\prime}+B^{\prime}\right)$. By 
Proposition 1.1

$$
m\left(A^{\prime}+B^{\prime}\right)=m\left(A^{\prime}+H\right)+m\left(B^{\prime}+H\right)-m(H),
$$

so $m(A)+m(B)=m(A+B)=m\left(A^{\prime}+H\right)+m\left(B^{\prime}+H\right)-m(H)$. Rearranging, we get

$$
m\left(A^{\prime}+H\right)-m(A)+m\left(B^{\prime}+H\right)-m(B)=m(H) .
$$

Let $A=\bigcup_{i=1}^{n} A_{i}$ and $B=\bigcup_{j=1}^{m} B_{j}$ be $H$-coset decompositions of $A$ and $B$. Since $A \subseteq A^{\prime}+H$ and $B \subseteq B^{\prime}+H$, (3.2) implies

$$
m\left(A_{i}\right)+m\left(B_{j}\right) \geq m(H)
$$

for each $i$ and $j$ (otherwise the left-hand side of (3.2) would be larger than $m(H)$ ). If the inequality (3.3) is strict for each pair $i$ and $j$, we have (E.1). Otherwise, take $i$ and $j$ so that equality holds in (3.2) and set $A_{0}=A_{i}, B_{0}=B_{j}$. If $A=A_{0}$ and $B=B_{0}$, then $(A, B)$ is complementary with respect to $H$. If not, we set $A_{1}=A \backslash A_{0}$ and $B_{1}=B \backslash B_{0}$, we verify (QP.1)-(QP.3) in Theorem 1.3. Equation (3.2) implies $A_{1} \sim A_{1}+H$ and $B_{1} \sim B_{1}+H$, so (QP.1) holds. If $A+B \neq A+B+H$, we conclude that $A_{0}+B_{0}+H$ is a unique expression element of $A+B+H$ in $G / H$ and we have (QP.2). (QP.3) now follows from $A+B \sim A+B+H$.

\section{EXAMPLES}

We list some examples of sur-critical pairs to show that each alternative in Theorem 1.3 can occur, and that none of the alternatives can be omitted. We do not attempt to exhaustively construct all possible sur-critical pairs.

4.1. Periodic pairs. If $F$ is a finite group, every sur-critical pair for $F$ satisfies $(\mathrm{P})$ and not $(\mathrm{K})$, and every nonextendible sur-critical pair satisfies $(\mathrm{P})$ but not $(\mathrm{E})$. A specific example with $F=\mathbb{Z} / 11 \mathbb{Z}$ is $A=\{0,1\}, B=\{0,3\}$, so that $A+B=$ $\{0,1,3,4\}$, and $(A, B)$ satisfies $(\mathrm{P})$ but not $(\mathrm{E})$ or $(\mathrm{K})$. Every periodic sur-critical pair $(A, B)$ for a compact group $G$ has the form $A=\phi^{-1}(C) \backslash N, B=\phi^{-1}(D) \backslash N$, where $(C, D)$ is a sur-critical pair for a finite group $F, \phi: G \rightarrow F$ is a continuous surjective homomorphism, and $m(N)=0$, so the periodic sur-critical pairs for an arbitrary compact group $G$ are classified in [4].

4.2. Extendible pairs. The following example satisfies $(\mathrm{E})$, but not $(\mathrm{P}),(\mathrm{K})$, or $(\mathrm{QP})$. Let $G=(\mathbb{Z} / 17 \mathbb{Z}) \times \mathbb{T}$ and let $A=\{1,3,5,7\} \times[0,0.8], B=\{0,2\} \times[0,0.9]$, so that $A+B=\{1,3,5,7,9\} \times \mathbb{T}$. Then $m(A)=3.2 / 17, m(B)=1.8 / 17$, and $m(A+B)=5 / 17$. Also $A+B=A^{\prime}+B^{\prime}$, where $A^{\prime}=\{1,3,5,7\} \times \mathbb{T}$ and $B^{\prime}=\{0,2\} \times \mathbb{T}$.

To find examples satisfying (E.1) (\$3.3), fix a pair of sets $A^{\prime}, B^{\prime} \subseteq G$ satisfying $m\left(A^{\prime}+B^{\prime}\right)<m\left(A^{\prime}\right)+m\left(B^{\prime}\right)$, and let $H=H\left(A^{\prime}+B^{\prime}\right)$. Choose any pair of subsets $A \subseteq A^{\prime}, B \subseteq B^{\prime}$ with $m(A)+m(B)=m\left(A^{\prime}+B^{\prime}\right)$, subject to the condition that $m((a+H) \cap A)+m((b+H) \cap B)>m(H)$ for all $a \in A^{\prime}$ and $b \in B^{\prime}$. Then $A+B=A^{\prime}+B^{\prime}$, so $m(A+B)=m(A)+m(B)$.

To find examples satisfying (E.2) but not (E.1), recall the construction in 93.1 Let $(A, B)$ satisfy conclusion (QP) of Theorem 1.3 with $m\left(A_{0}\right)+m\left(B_{0}\right)=m(K)$, but $A_{0}+B_{0} \neq A_{0}+B_{0}+K$. To form a specific example of this construction with $G=(\mathbb{Z} / 15 \mathbb{Z}) \times \mathbb{T}$, let $A=(\{1,3,5\} \times \mathbb{T}) \cup(\{7\} \times S)$, where $S \subseteq \mathbb{T}$ is any measurable set, and let $B=(\{0,2\} \times \mathbb{T}) \cup\left(\{4\} \times\left(-S^{c}\right)\right)$. This example satisfies (E) and (QP) but not $(\mathrm{P})$ or $(\mathrm{K})$. 
4.3. Pairs arising from $\mathbb{T}$. Let $G$ be a compact group which is not totally disconnected. Let $(A, B)$ have the form $A=\chi^{-1}(I), B=\chi^{-1}(J)$, where $H \leqslant G$ is a compact open subgroup, $\chi: H \rightarrow \mathbb{T}$ is a continuous surjective homomorphism, $I, J \subseteq \mathbb{T}$ are intervals, and $m_{\mathbb{T}}(I)+m_{\mathbb{T}}(J)<1$. One can verify that $(A, B)$ is a sur-critical pair satisfying $(\mathrm{K})$ but not $(\mathrm{P}),(\mathrm{E})$, or $(\mathrm{QP})$.

4.4. A quasi-periodic pair. This example will satisfy (QP) but not (P), (E), or $(\mathrm{K})$.

Let $G=\mathbb{Z}_{7}$, the 7 -adic integers with the usual topology, and consider $\mathbb{Z}$ as a subset of $\mathbb{Z}_{7}$ in the usual way. Define the set $C \subseteq \mathbb{Z}$ by

$$
C:=(\{0,1\}+7 \mathbb{Z}) \cup(2+7((\{0,1\}+7 \mathbb{Z}) \cup(2+7(\cdots)))),
$$

so that $C=(\{0,1\}+7 \mathbb{Z}) \cup(2+7 C)$. Let $A$ be the closure of $C$ in $\mathbb{Z}_{7}$, and let $B=A$. Then $m(A)=m(B)=\sum_{n=1}^{\infty} \frac{2}{7^{n}}=1 / 3$. Note that $A$ has a quasi-periodic decomposition $A_{1} \cup A_{0}$ where $A_{1}=\overline{\{0,1\}+7 \mathbb{Z}}, A_{0}=A \backslash A_{1}$. The sumset $A+B$ is the closure of $C+C$ in $\mathbb{Z}_{7}$. Note that

$$
C+C=(\{0,1,2,3\}+7 \mathbb{Z}) \cup(4+7((\{0,1,2,3\}+7 \mathbb{Z}) \cup(4+7(\cdots)))),
$$

so $m(A+B)=2 / 3=m(A)+m(B)$. From (4.1) one can check that $H(A+B)=\{0\}$, so (P) and (E) fail. Since $G$ is totally disconnected, $(\mathrm{K})$ cannot hold.

4.5. More quasi-periodic pairs. Let $G^{\prime}$ be an infinite compact abelian group with Haar measure $m^{\prime}$, and let $G=(\mathbb{Z} / 4 \mathbb{Z}) \times G^{\prime}$. Fix sets $A_{0}^{\prime}, B_{0}^{\prime} \subseteq G^{\prime}$ satisfying $m_{*}^{\prime}\left(A_{0}^{\prime}+B_{0}^{\prime}\right)=m^{\prime}\left(A_{0}^{\prime}\right)+m_{0}\left(B_{0}^{\prime}\right)$, let $A_{1}=\{0\} \times G^{\prime}, B_{1}=\{0\} \times G^{\prime}$, and let $A_{0}=\{1\} \times A_{0}^{\prime}, B_{0}=\{1\} \times B_{0}^{\prime}$. Let $A=A_{1} \cup A_{0}, B=B_{1} \cup B_{0}$. Then $m_{*}(A+B)=$ $m(A)+m(B)$, but depending on our choice of $A_{0}$ and $B_{0}, A+B$ may not be measurable. If $A_{0}^{\prime}$ is a singleton, then $B_{0}^{\prime}$ may be an arbitrary measurable subset of $G^{\prime}$.

\section{LEMMAS}

We fix a compact abelian group $G$ with Haar measure $m$. Unless stated otherwise, sets $A$ and $B$ are assumed to be subsets of $G$.

5.1. Consequences of Proposition 1.1. We need Lemma 5.1 and Corollary 5.2 in many subsequent proofs. See 2.1 .6 for notation.

Lemma 5.1. If $m_{*}(A+B)<m(A)+m(B)$, then for all $a \in A$ and all $b \in B$,

$$
\begin{aligned}
& m((a+H) \cap A)+m_{*}(A+B) \geq m(A)+m(B), \\
& m((b+H) \cap B)+m_{*}(A+B) \geq m(A)+m(B),
\end{aligned}
$$

where $H=H(A+B)$.

Proof. We prove (5.1). Write $m(A)=\sum_{i=1}^{n} m\left(A_{i}\right)$, where $A=\bigcup_{i=1}^{n} A_{i}$ is an $H$ coset decomposition of $A$. Fix $a \in A$, and let $j$ be such that $(a+H) \cap A=A_{j}$. Then $m\left(A_{j}\right)=m(A)-\sum_{i \neq j} m\left(A_{i}\right)$, while $m(A+H)=\sum_{i=1}^{n} m(H)$. Now Proposition 
1.1 implies

$$
\begin{aligned}
m\left(A_{j}\right)+m_{*}(A+B) & =\left(m(A)-\sum_{i \neq j} m\left(A_{i}\right)\right)+m(A+H)+m(B+H)-m(H) \\
= & \left(m(A)-\sum_{i \neq j} m\left(A_{i}\right)\right)+\left(\sum_{i} m(H)\right)+m(B+H)-m(H) \\
= & m(A)+\left(\sum_{i \neq j} m(H)-m\left(A_{i}\right)\right)+m(H)+m(B+H)-m(H) \\
\geq & m(A)+m(B+H) \\
\geq & m(A)+m(B) .
\end{aligned}
$$

Equation (5.2) follows by symmetry.

We write $C \subset_{m} D$ below to mean $m(C \backslash D)=0$.

Corollary 5.2. If $m_{*}(A+B)<m(A)+m(B)$, let $H:=H(A+B)$. Then

$$
\begin{aligned}
& \left\{z \in G: z+B \subset_{m} A+B\right\}=\{z \in G: z+B \subseteq A+B\}=A+H, \\
& \left\{z \in G: A+z \subset_{m} A+B\right\}=\{z \in G: A+z \subseteq A+B\}=B+H .
\end{aligned}
$$

Proof. For the first equality in (5.3), note that $z+B \subset_{m} A+B$ implies $z+B \subseteq$ $A+B$, by Lemma 5.1 and the fact that $A+B$ is a union of cosets of $H$. For the second equality in (5.3), we argue by contradiction. Fix $z \in G$ such that $z+B \subseteq A+B$, but $z \notin A+H$. Let $A^{\prime}=A \cup(z+H)$. Then $A^{\prime}+B=A+B$, so that $H\left(A^{\prime}+B\right)=H(A+B)$, while $m\left(A^{\prime}+H\right)=m(A+H)+m(H)$. Applying Proposition 1.1 to $\left(A^{\prime}, B\right)$ yields

$$
\begin{aligned}
m\left(A^{\prime}+B\right) & =m\left(A^{\prime}+H\right)+m(B+H)-m(H) \\
& =m(A+H)+m(B+H),
\end{aligned}
$$

which contradicts the assumption that $m_{*}(A+B)<m(A)+m(B)$. Equation (5.4) follows by symmetry.

Lemma 5.3. If $m_{*}(A+B)<m(A)+m(B)$ and $H:=H(A+B)$, then for all $a \in A$ and $b \in B$,

$$
m((a+H) \cap A)+m((b+H) \cap B)>m(H) .
$$

Proof. If inequality (5.5) fails, then $m(A+H)+m(B+H) \geq m(A)+m(B)+$ $m(H)$, so inequality (1.2) in Proposition 1.1 implies $m_{*}(A+B) \geq m(A)+m(B)$, contradicting the hypothesis.

In the next corollary we use the elementary fact that if $G$ is compact, and $C$, $D \subseteq G$ have $m(C)+m(D)>1$, then $C+D=G$. This fact follows from the observation that $t \in C+D$ if and only if $(t-C) \cap D \neq \varnothing$, while $t-C$ and $D$ cannot be disjoint if $m(C)+m(D)>m(G)$. Consequently, if $H \leqslant G$ is a compact open subgroup and $C, D \subset G$ are each contained in a coset of $H$ while $m(C)+m(D)>m(H)$, then $C+D=C+D+H$.

Corollary 5.4. If $m_{*}(A+B)<m(A)+m(B)$, then $A-B$ is periodic with period $H:=H(A+B)$. 
Proof. Let $A=\bigcup_{i=1}^{n} A_{i}$ and $B=\bigcup_{i=1}^{m} B_{i}$ be $H$-coset decompositions of $A$ and $B$, so that $A-B=\bigcup_{i, j} A_{i}-B_{j}$. Inequality (5.5) implies $m\left(A_{i}\right)+m\left(B_{j}\right)>m(H)$ for each $i$ and $j$, so $A_{i}-B_{j}=A_{i}-B_{j}+H$ for each $i$ and $j$, implying $A-B=$ $A-B+H$.

5.2. Reducible pairs. We now dispense with the case of Theorem 1.3 where $(A, B)$ is reducible $(\$ 2.2 .4)$. For the next two lemmas and the following corollary, we assume that $m(A)>0, m(B)>0$, and $(A, B)$ satisfies $m_{*}(A+B)=m(A)+m(B)$.

Lemma 5.5. If $A^{\prime} \subseteq A$ is such that $m\left(A^{\prime}\right)=m(A)$ and $m_{*}\left(A^{\prime}+B\right)<m_{*}(A+B)$, then $A^{\prime}+H\left(A^{\prime}+B\right) \subset_{m} A$ and at least one of the following holds:

(i) $(A, B)$ satisfies (QP) of Theorem 1.3 , with $K=H:=H\left(A^{\prime}+B\right)$, $A_{1}=A \cap\left(A^{\prime}+H\right)$, and $A_{0}=A \backslash A_{1}$, so that $m\left(A_{1}\right)=m(A)$ and $m\left(A_{0}\right)=0$.

(ii) $B \sim B+H\left(A^{\prime}+B\right)$.

If $(A, B)$ is nonextendible, then (i) holds.

Proof. If $A, B, A^{\prime}$ and $H$ are as in the hypothesis, Proposition 1.1 implies $H$ is compact and open and $A^{\prime}+B$ is a union of cosets of $H$. Corollary 5.2 implies that whenever $a_{0} \in A$ is such that $a_{0}+B \nsubseteq A^{\prime}+B$, there is a $b_{0} \in B$ such that

$$
m\left(\left(a_{0}+B_{0}\right) \backslash\left(A^{\prime}+B\right)\right)>0,
$$

where $B_{0}:=\left(b_{0}+H\right) \cap B$. Fix $a_{0} \in A, b_{0} \in B$, and $B_{0}$ satisfying (5.6), and let $B_{1}=B \backslash B_{0}$. Lemma 5.1 and the hypothesis $m\left(A^{\prime}\right)=m(A)$ imply

$$
m\left(B_{0}\right)+m\left(A^{\prime}+B\right) \geq m\left(A^{\prime}\right)+m(B)=m_{*}(A+B) .
$$

Claim 1. $A^{\prime}+H \subset_{m} A$ and $B_{1}+H \subset_{m} B$.

Proof of Claim 1. By the definition of $B_{0}$ and the fact that $A^{\prime}+B=A^{\prime}+B+H$, $A^{\prime}+B$ is disjoint from $a_{0}+B_{0}$. Using (5.7) we write

$$
m_{*}(A+B)=m\left(A^{\prime}+B\right)+m\left(B_{0}\right) .
$$

Then

$$
\begin{aligned}
m\left(A^{\prime}\right)+m(B) & =m_{*}(A+B) \\
& =m\left(A^{\prime}+B\right)+m\left(B_{0}\right) \\
& =m\left(A^{\prime}+H\right)+m(B+H)-m(H)+m\left(B_{0}\right) \\
& =m\left(A^{\prime}+H\right)+m\left(B_{1}+H\right)+m\left(B_{0}\right),
\end{aligned}
$$

where the second line is (5.8), the third line is from Proposition 1.1 and the fourth line is due to $B=B_{1} \cup B_{0}$ being a disjoint union and $B_{0}$ being contained in a coset of $H$. Subtracting $m\left(A^{\prime}\right)+m(B)$ from the first and last lines of (5.9), we find

$$
\left[m\left(A^{\prime}+H\right)-m\left(A^{\prime}\right)\right]+\left[m\left(B_{1}+H\right)+m\left(B_{0}\right)-m(B)\right]=0 .
$$

Each bracketed summand in (5.10) is nonnegative, so

$$
m\left(A^{\prime}\right)=m\left(A^{\prime}+H\right)
$$

and

$$
m(B)=m\left(B_{1}+H\right)+m\left(B_{0}\right) .
$$

Since $\left(B_{1}+H\right) \cap B_{0}=\varnothing$ and $B=B_{1} \cup B_{0}$, (5.11) and (5.12) prove the claim.

We proceed based on whether $m\left(B_{0}\right)<m(H)$. 
Case 1. $m\left(B_{0}\right)=m(H)$. In this case Claim 1 implies $B \sim B+H$, and we conclude (ii) in the statement of the lemma.

Case 2. $m\left(B_{0}\right)<m(H)$. Let $A_{0}:=A \backslash\left(A^{\prime}+H\right)$. We will show conclusion (i) of the lemma holds.

Claim 2. $A_{0}$ is contained in a coset of $H$ and $A_{0}+B_{0}+H$ is a unique expression element of $A+B+H$ in $G / H$.

Proof of Claim 2. Observe that $A+B_{1} \subseteq A^{\prime}+B$, since otherwise Claim 1 implies $m_{*}(A+B) \geq m\left(A^{\prime}+B\right)+m(H)>m\left(A^{\prime}+B\right)+m\left(B_{0}\right)$, contradicting (5.8). Furthermore, $A^{\prime}+H$ is the set $\left\{z \in G: z+B \subset_{m} A^{\prime}+B\right\}$, by Corollary 5.2 . Thus, $A_{0}$ is the set of $a \in A$ such that $a+B_{0} \nsubseteq A^{\prime}+B$. Now if $a, a^{\prime} \in A_{0}$, then $a+B_{0} \sim a^{\prime}+B_{0}$, by (5.8). Since $B_{0}$ is contained in a coset of $H$ and $m\left(B_{0}\right)>0$, the similarity $a+B_{0} \sim a^{\prime}+B_{0}$ implies $a-a^{\prime} \in H$. We conclude that $A_{0}$ is contained in a coset of $H$. Now the containment $A+B_{1} \subseteq A^{\prime}+B$ implies $A_{0}+B_{0}+H$ is a unique expression element of $A+B+H$ in $G / H$, as claimed.

Writing $A_{1}=A \cap\left(A^{\prime}+H\right)$, and maintaining $A_{0}, B_{1}$, and $B_{0}$ as defined above, the partition $A=A_{1} \cup A_{0}$ is a quasi-periodic decomposition with quasi-period $H$, while $B_{1} \sim B_{1}+H$, and $B_{0}$ is contained in a coset of $H$ (possibly $B_{1}=\varnothing$ ), so we have verified that (QP.1) and (QP.2) hold. To verify (QP.3), consider the partition $A+B=\left(A_{1}+B\right) \cup\left(A_{0}+B_{0}\right)$, so that (5.8) implies $m_{*}\left(A_{0}+B_{0}\right)=m\left(B_{0}\right)$. Since $m\left(A_{0}\right)=0$, we then have $m_{*}\left(A_{0}+B_{0}\right)=m\left(A_{0}\right)+m\left(B_{0}\right)$. This concludes the analysis of Case 2 .

Now suppose $(A, B)$ is nonextendible. If Case 2 holds, we have conclusion (i), as desired. If Case 1 holds we derive a contradiction: from $B+H \sim B$ and the nonextendibility of $(A, B)$ we find $A \sim A+H$. Since $H$ is compact and open, the similarity $A \sim A+H$ implies $m\left(A^{\prime \prime}+B\right)=m_{*}(A+B)$ whenever $A^{\prime \prime} \subseteq A$ has $m\left(A^{\prime \prime}\right)=m(A)$, contradicting the hypothesis of the lemma.

Lemma 5.6. If $(A, B)$ is reducible and nonextendible, then $(A, B)$ has a quasiperiodic decomposition $A=A_{1} \cup A_{0}, B=B_{1} \cup B_{0}$ satisfying conclusion (QP) of Theorem 1.3, and at least one of $m\left(A_{0}\right)=0$ or $m\left(B_{0}\right)=0$.

Proof. Let $A^{\prime} \subseteq A$ and $B^{\prime} \subseteq B$ be such that $m\left(A^{\prime}\right)+m\left(B^{\prime}\right)=m(A)+m(B)$ and $m_{*}\left(A^{\prime}+B^{\prime}\right)<m_{*}(A+B)$. Write $H:=H\left(A^{\prime}+B^{\prime}\right)$, and note that $H$ is compact and open, by Proposition 1.1. We consider two cases.

Case 1. $m_{*}\left(A^{\prime}+B\right)<m_{*}(A+B)$ or $m_{*}\left(A+B^{\prime}\right)<m_{*}(A+B)$. We apply Lemma 5.5 and we are done.

Case 2. $m_{*}\left(A^{\prime}+B\right)=m_{*}\left(A+B^{\prime}\right)=m_{*}(A+B)$. In this case Lemma 5.5 applies to the pairs $\left(B, A^{\prime}\right)$ and $\left(A, B^{\prime}\right)$, so

$$
B^{\prime}+H \sim B \text { and } A^{\prime}+H \sim A .
$$

We claim that $A_{0}:=A \backslash\left(A^{\prime}+H\right)$ is contained in a coset of $H$. Let $t$ be the number of cosets of $H$ occupied by $A \backslash\left(A^{\prime}+H\right)$, so that

$$
m(A+H)-m\left(A^{\prime}\right)=t \cdot m(H) .
$$

We aim to show that $t=1$, so that $A_{0}$ is contained in a coset of $H$. We have $t>0$, as the hypothesis of Case 2 implies $A+H \neq A^{\prime}+H$. 
Consider the pair $\left(A+H, B^{\prime}\right)$. By (5.13),$A+H+B^{\prime} \sim A+B^{\prime} \sim A+B$. Then

$$
\begin{array}{rlrl}
m\left((A+H)+B^{\prime}\right) & =m_{*}(A+B) & & \\
& =m(A)+m(B) & & \text { (by hypothes } \\
& =m\left(A^{\prime}+H\right)+m\left(B^{\prime}\right) & & (\text { by }[5.13) \\
& =m(A+H)-t \cdot m(H)+m\left(B^{\prime}\right) & & (\text { by (5.14) }) \\
& <m(A+H)+m\left(B^{\prime}\right) . &
\end{array}
$$

Applying Proposition 1.1 to the pair $\left(A+H, B^{\prime}\right)$ we get that $K:=H\left((A+H)+B^{\prime}\right)$ is compact and open,

$$
A+H+B^{\prime}+K=A+H+B^{\prime}
$$

and

$$
m\left(A+H+B^{\prime}\right)=m(A+H+K)+m\left(B^{\prime}+K\right)-m(K) .
$$

We want to show $K \leqslant H$. The nonextendibility of $(A, B)$, (5.13), and (5.16) together imply $B^{\prime}+K \subset_{m} B$. It follows that $B^{\prime}+K \subset_{m} E$ whenever $E \sim B$. Consequently $B^{\prime}+K \subset_{m} B^{\prime}$, and we conclude $A^{\prime}+B^{\prime} \sim A^{\prime}+B^{\prime}+K$, so $K \leqslant H$ by the definition of $H$. Now (5.17) becomes

$$
m\left(A+H+B^{\prime}\right)=m(A+H)+m\left(B^{\prime}\right)-m(K) .
$$

Comparing (5.18) with the fourth line of (5.15), we get $t \cdot m(H)=m(K)$. This implies $t=1$, since $K \subseteq H$ and $m(K)>0$.

We have shown that $A_{0}$ is contained in a coset of $H$, so $A_{1}:=A \backslash A_{0}$ gives the desired quasi-periodic decomposition of $A$. Reversing the roles of $A$ and $B$, we find the corresponding quasi-periodic decomposition of $B: B_{0}=B \backslash\left(B^{\prime}+H\right)$, $B_{1}=B \backslash B_{0}$. We now show that $A_{1} \cup A_{0}$ and $B_{1} \cup B_{0}$ satisfy (QP.2) and (QP.3).

Observe that $m\left(A_{0}\right)=m\left(B_{0}\right)=0$, by our choice of $A_{0}$ and $B_{0}$. Nonextendibility of $(A, B)$ implies $A_{0}+B_{0}+H$ is a unique expression element of $A+B+H$ in $G / H$. To see that $m_{*}\left(A_{0}+B_{0}\right)=0$, write $A+B$ as $\left(A_{1}+B\right) \cup\left(A_{0}+B_{0}\right)$, so that $m_{*}(A+B)=m\left(A_{1}+B\right)+m_{*}\left(A_{0}+B_{0}\right)$. By the hypothesis of Case 2, we have $m_{*}\left(A_{1}+B\right)=m_{*}(A+B)$, so $m_{*}\left(A_{0}+B_{0}\right)=0=m\left(A_{0}\right)+m\left(B_{0}\right)$.

Corollary 5.7. If $(A, B)$ is reducible and $m(H(A+B))=0$, then $(A, B)$ satisfies conclusion (QP) of Theorem 1.3 with at least one of $m\left(A_{0}\right)=0$ or $m\left(B_{0}\right)=0$.

Proof. If $m(H(A+B))=0$, then $(A, B)$ is nonextendible, by Proposition 1.1. The conclusion now follows from Lemma 5.6 .

5.3. Auxiliary lemmas. The next two lemmas handle technicalities arising repeatedly in the next subsection. We continue to assume $(A, B)$ satisfies the hypotheses of Theorem 1.3 .

Lemma 5.8. Suppose $(A, B)$ is irreducible and nonextendible. Assume further that $H:=H(\tilde{A}+\tilde{B})$ has positive Haar measure for some $\tilde{A} \subseteq A, \tilde{B} \subseteq B$ such that $\tilde{A}+\tilde{B}$ is measurable, $m(\tilde{A})=m(A)$ and $m(\tilde{B})=m(B)$. Then $A$ and $B$ are periodic with period $H$, or $(A, B)$ satisfies $(\mathrm{QP})$ of Theorem 1.3 with $K=H$.

Proof. Let $\delta=m(H)$. Since $m(H)>0$, we have $m\left((\tilde{A}+\tilde{B}) \cap H_{i}\right) \in\{0, \delta\}$ for every coset $H_{i}$ of $H$ (cf. 2.2 .1 ). Irreducibility of $(A, B)$ then implies

$$
m_{*}\left((A+B) \cap H_{i}\right) \in\{0, \delta\} \text { for every coset } H_{i} \text { of } H \text {. }
$$


Combining (5.19) with the nonextendibility of $(A, B)$, we have $m\left(A \cap H_{i}\right) \in\{0, \delta\}$ and $m\left(B \cap H_{i}\right) \in\{0, \delta\}$ for every coset $H_{i}$ of $H$. Now by the irreducibility of $(A, B)$, there are sets $A^{\prime} \subseteq A, B^{\prime} \subseteq B$ such that

$$
A \sim A^{\prime}+H \sim A^{\prime} \text { and } B \sim B^{\prime}+H \sim B^{\prime},
$$

$A^{\prime}+B^{\prime}$ is measurable, and $m_{*}(A+B)=m\left(A^{\prime}+B^{\prime}\right)$. Setting $A_{0}:=A \backslash\left(A^{\prime}+H\right)$, we claim $A_{0}$ is contained in a coset of $H$. To prove this claim, assume $A_{0}$ is nonempty, let $a \in A_{0}$, and consider the pair $\left(A^{\prime} \cup(a+H), B^{\prime}\right)$. Write $C$ for $A^{\prime} \cup(a+H)$, so $m(C)=m\left(A^{\prime}\right)+m(H)$. By (5.20) we have $a+B^{\prime}+H \subset_{m} A+B$, so $C+B^{\prime} \subset_{m} A+B$. The irreducibility of $(A, B)$ then implies

$$
C+B^{\prime} \sim \tilde{A}+\tilde{B}
$$

so that $H\left(C+B^{\prime}\right)=H(\tilde{A}+\tilde{B})$. We also have

$$
m\left(C+B^{\prime}\right)=m_{*}(A+B)=m(A)+m(B)<m(C)+m\left(B^{\prime}\right),
$$

so we may apply Corollary 5.2 to the pair $\left(C, B^{\prime}\right)$ and find

$$
C+H=\left\{z \in G: z+B^{\prime} \subset_{m} C+B^{\prime}\right\} .
$$

By (5.21), the right-hand side of (5.22) is also $\left\{z \in G: z+B^{\prime} \subset_{m} \tilde{A}+\tilde{B}\right\}$, which does not depend on $a \in A_{0}$. Hence, $\left(A^{\prime}+H\right) \cup(a+H)$ does not depend on the choice of $a \in A_{0}$. This means that $A_{0}$ is contained in a coset of $H$, as claimed.

If $A_{0}=\varnothing$, then $B \sim B+H$ by the irreducibility and nonextendibility of $(A, B)$, so $A$ and $B$ are periodic with period $H$, and we are done. If $A_{0}$ is nonempty, we will show that $(A, B)$ satisfies $(\mathrm{QP})$. Set $A_{1}=A \backslash A_{0}$ to get the desired quasi-periodic decomposition $A_{1} \cup A_{0}$ of $A$ with $m\left(A_{0}\right)=0$. Reversing the roles of $A$ and $B$ in the previous paragraph, we find that either $B$ is $H$-periodic or has a quasi-periodic decomposition $B_{1} \cup B_{0}$ with respect to $H$, where $m\left(B_{0}\right)=0$. The nonextendibility of $(A, B)$ implies $B_{0}$ is nonempty, and also implies $A_{0}+B_{0}+H$ is a unique expression element of $A+B+H$ in $G / H$. Now (5.19) and the nonextendibility of $(A, B)$ imply $m_{*}\left(A_{0}+B_{0}\right)=0=m\left(A_{0}\right)+m\left(B_{0}\right)$.

Lemma 5.9. Let $\left(A^{\prime}, B^{\prime}\right)$ be an irreducible nonextendible sur-critical pair satisfying (QP) of Theorem [1.3 with decomposition $A^{\prime}=A_{1}^{\prime} \cup A_{0}^{\prime}, B^{\prime}=B_{1}^{\prime} \cup B_{0}^{\prime}$, such that $m\left(A_{0}^{\prime}\right)>0$ and $m\left(B_{0}^{\prime}\right)>0$. If $(A, B)$ is another irreducible nonextendible surcritical pair such that $A \sim A^{\prime}$ and $B \sim B^{\prime}$, then $(A, B)$ also satisfies (QP).

Proof. We may assume $A^{\prime} \subseteq A$ and $B^{\prime} \subseteq B$, since we can replace $A^{\prime}$ and $B^{\prime}$ with $A^{\prime} \cap A$ and $B^{\prime} \cap B$ while maintaining the hypotheses of the lemma - this follows from the irreducibility of $(A, B)$ and $\left(A^{\prime}, B^{\prime}\right)$. Let $K$ be the quasi-period of $\left(A^{\prime}, B^{\prime}\right)$ from conclusion (QP). Assume, without loss of generality, that $A_{1}^{\prime} \neq \varnothing$. Define $A_{0}:=A \cap\left(A_{0}^{\prime}+K\right)$ and $A_{1}:=A \backslash A_{0}$. We will show that $A_{1} \sim A_{1}+K$.

Let $a \in A_{1}$. We aim to show that $a+K \subseteq A_{1}^{\prime}+K$. Irreducibility of $(A, B)$ implies $a+B^{\prime} \subset_{m} A^{\prime}+B^{\prime}$. Since $a \notin A_{0}^{\prime}+K$, we have $a+B^{\prime} \subset_{m}\left(A_{1}^{\prime}+B^{\prime}\right) \cup\left(A+B_{1}^{\prime}\right)$, so that $a+B^{\prime}+K \subset_{m} A^{\prime}+B^{\prime}$. Then $a+K \subset_{m} A^{\prime}$ by the nonextendibility of $\left(A^{\prime}, B^{\prime}\right)$. Consequently, $a+K \subseteq A_{1}^{\prime}+K$, and we find $A_{1}+K=A_{1}^{\prime}+K$. The similarity $A \sim A^{\prime}$ then implies $A_{1} \sim A_{1}^{\prime}$, and $A=A_{1} \cup A_{0}$ is a quasi-periodic decomposition. Reversing the roles of $A$ and $B$, we define $B_{0}=B \cap\left(B_{0}^{\prime}+K\right)$ and $B_{1}=B \backslash B_{0}$. Now it is routine to verify that $(A, B)$ also satisfies $(\mathrm{QP})$ of Theorem 1.3 . 


\subsection{Measurability and trivializing $H(A+B)$.}

Lemma 5.10. If Theorem 1.3 holds under the additional assumption that $A+B$ is measurable, then Theorem 1.3 holds in general.

Proof. We first list cases where we obtain the conclusion of Theorem 1.3 without assuming $A+B$ is measurable. Excluding those cases, we then show how to deduce the conclusion of Theorem 1.3 from the special case where $A+B$ is assumed to be measurable.

If $(A, B)$ is extendible, then $(A, B)$ satisfies conclusion (E) of Theorem 1.3. If $(A, B)$ is nonextendible and reducible, then $(A, B)$ satisfies conclusion (QP) of Theorem 1.3 by Lemma 5.6. We may therefore assume that $(A, B)$ is nonextendible and irreducible. Let $A^{\prime} \subseteq A$ and $B^{\prime} \subseteq B$ be countable unions of compact sets with $m\left(A^{\prime}\right)=m(A)$ and $m\left(B^{\prime}\right)=m(B)$, so that $A^{\prime}+B^{\prime}$ is measurable.

Irreducibility of $(A, B)$ implies $m\left(A^{\prime}+B^{\prime}\right)=m_{*}(A+B)$, so $\left(A^{\prime}, B^{\prime}\right)$ satisfies the hypotheses of Theorem 1.3. If $\left(A^{\prime}, B^{\prime}\right)$ satisfies conclusion (P) or conclusion (E) of Theorem 1.3, then $m\left(H\left(A^{\prime}+B^{\prime}\right)\right)>0$ by Proposition 1.1. We apply Lemma 5.8 and conclude that $(A, B)$ satisfies one of conclusion (P) or (QP). If $\left(A^{\prime}, B^{\prime}\right)$ satisfies $(\mathrm{K})$, so that $A^{\prime} \sim a+\chi^{-1}(I)$ and $B^{\prime} \sim b+\chi^{-1}(J)$, where $I, J \subseteq \mathbb{T}$ are intervals and $\chi: K \rightarrow \mathbb{T}$ is a continuous surjective homomorphism from a compact open subgroup $K \leqslant G$, then a routine argument (2.1.1) shows that $A$ and $B$ must be contained in $a+\chi^{-1}(I)$, and $b+\chi^{-1}(J)$, respectively. We conclude that $(A, B)$ satisfies $(\mathrm{K})$.

The only remaining possibility is that $\left(A^{\prime}, B^{\prime}\right)$ satisfies $(\mathrm{QP})$, but not $(\mathrm{P})$ or (E). Then $\left(A^{\prime}, B^{\prime}\right)$ is nonextendible, while irreducibility of $(A, B)$ implies that of $\left(A^{\prime}, B^{\prime}\right)$. Writing $A^{\prime}=A_{1}^{\prime} \cup A_{0}^{\prime}$ and $B^{\prime}=B_{1}^{\prime} \cup B_{0}^{\prime}$ for the decomposition of $\left(A^{\prime}, B^{\prime}\right)$ in (QP) and $K$ for the corresponding subgroup, we consider different cases based on the measures of $A_{0}^{\prime}$ and $B_{0}^{\prime}$.

If $m\left(A_{0}^{\prime}\right)>0$ and $m\left(B_{0}^{\prime}\right)>0$, Lemma 5.9 implies $(A, B)$ satisfies (QP).

If $m\left(A_{0}^{\prime}\right)=0$ and $m\left(B_{0}^{\prime}\right)>0$, or vice versa, we derive a contradiction. Under these conditions, $\left(A^{\prime}, B^{\prime}\right)$ is reducible, since $A_{0}^{\prime}+B_{0}^{\prime}+K$ is a unique expression element of $A^{\prime}+B^{\prime}+K$ in $G / K$. Reducibility of $\left(A^{\prime}, B^{\prime}\right)$ contradicts our present assumptions.

If $m\left(A_{0}^{\prime}\right)=m\left(B_{0}^{\prime}\right)=0$, the irreducibility of $(A, B)$ implies $A+B \sim A_{1}^{\prime}+B_{1}^{\prime}$. Since $H\left(A_{1}^{\prime}+B_{1}^{\prime}\right)$ has positive Haar measure, we apply Lemma 5.8 with $\tilde{A}=A_{1}^{\prime}$, $\tilde{B}=B_{1}^{\prime}$ and conclude that $(A, B)$ satisfies $(\mathrm{P})$ or $(\mathrm{QP})$ of Theorem 1.3 .

The next lemma is a rephrasing of Lemma 7 of [12] we include a proof for completeness. Under the standing assumption that $G$ is compact, the group $H(S)$ below is compact, so the convention that Haar measure is normalized applies: $m_{H(S)}(H(S))=1$.

Lemma 5.11. If $S \subseteq G$ is measurable, set $H:=H(S)$ and let

$$
E:=\left\{x \in G: 0<m_{H}(S-x)<1 \text { or } H \cap(S-x) \text { is not } m_{H} \text {-measurable }\right\} .
$$

Then $m(E)=0$. 
Proof. Consider the integrals

$$
\begin{aligned}
I_{1} & :=\iint 1_{S}(x-z) 1_{S}(x) d m_{H}(z) d m(x), \\
I_{2} & :=\iint 1_{S}(x-z) 1_{S}(x) d m(x) d m_{H}(z) .
\end{aligned}
$$

By Proposition 2.1] these integrals are well defined, and $I_{1}$ can be computed as

$$
\iint 1_{S}(x-z) d m_{H}(z) 1_{S}(x) d m(x)=\int m_{H}(S-x) 1_{S}(x) d m,
$$

while $I_{2}=\int m((S+z) \cap S) d m_{H}(z)=m(S)$. By Fubini's theorem, $I_{1}=I_{2}$, meaning $\int m_{H}(S-x) 1_{S}(x) d m(x)=m(S)$. Since $0 \leq m_{H}(S-x) \leq 1$, the last equation implies

$$
m_{H}(S-x)=1 \text { for } m \text {-almost every } x \in S \text {. }
$$

Replacing $S$ with $S^{c}$, (5.23) implies $m_{H}(S-x)=0$ for $m$-almost every $x \in S^{c}$. The assertion follows.

Lemma 5.12. If $(A, B)$ is irreducible and satisfies the hypotheses of Theorem 1.3 , $A+B$ is measurable, and $m(H(A+B))=0$, then there are measurable sets $A^{\prime} \subseteq A$, $B^{\prime} \subseteq B$ such that $m\left(A^{\prime}\right)=m(A), m\left(B^{\prime}\right)=m(B), A^{\prime} \sim A^{\prime}+H, B^{\prime} \sim B^{\prime}+H$, and $A^{\prime}+B^{\prime}+H \sim A^{\prime}+B^{\prime} \sim A+B$, where $H:=H(A+B)$.

Proof. Let

$$
S_{1}:=\left\{x \in G: m_{H}(A-x)>0\right\}, \quad S_{2}:=\left\{x \in G: m_{H}(B-x)>0\right\} .
$$

By Proposition 2.1, $S_{1}$ and $S_{2}$ are measurable. Let $A^{\prime \prime}=A \cap S_{1}, B^{\prime \prime}=B \cap S_{2}$; Proposition 2.1 implies $m\left(A^{\prime \prime}\right)=m(A)$ and $m\left(B^{\prime \prime}\right)=m(B)$. Let $A^{\prime} \subseteq A^{\prime \prime}$ and $B^{\prime} \subseteq B^{\prime \prime}$ be countable unions of compact sets having $m\left(A^{\prime}\right)=m(A)$ and $m\left(B^{\prime}\right)=$ $m(B)$. The irreducibility of $(A, B)$ implies $A^{\prime}+B^{\prime} \sim A^{\prime \prime}+B^{\prime \prime} \sim A+B$, so that $H\left(A^{\prime}+B^{\prime}\right)=H$. By Lemma 5.11, the set

$E=\left\{x \in G: 0<m_{H}(A+B-x)<1\right.$ or $H \cap(A+B-x)$ is not $m_{H}$-measurable $\}$ has $m_{G}(E)=0$. By Lemma 5.11 and the definitions of $S_{A}, S_{B}$, and $E$,

$$
m_{H}\left(A^{\prime}+B^{\prime}+H-x\right) \leq m_{H}\left(A^{\prime \prime}+B^{\prime \prime}-x\right)=1
$$

whenever $x \notin E$ and $\left(A^{\prime \prime}+B^{\prime \prime}-x\right) \cap H$ is measurable and nonempty. Since $A^{\prime}$ and $B^{\prime}$ are countable unions of compact sets and $H$ is compact, $A^{\prime}+B^{\prime}+H$ is measurable. We now show $A^{\prime}+B^{\prime}+H \sim A^{\prime}+B^{\prime}$. By Proposition 2.1 and (5.24), then

$$
\begin{aligned}
m\left(A^{\prime}+B^{\prime}+H\right) & =\int m_{H}\left(A^{\prime}+B^{\prime}+H-x\right) d m(x) \\
& =\int_{E^{c}} m_{H}\left(A^{\prime}+B^{\prime}+H-x\right) d m(x) \\
& \leq \int_{E^{c}} m_{H}\left(A^{\prime \prime}+B^{\prime \prime}-x\right) d m(x) \\
& =m\left(A^{\prime \prime}+B^{\prime \prime}\right) .
\end{aligned}
$$

Thus $m\left(A^{\prime}+B^{\prime}+H\right) \leq m\left(A^{\prime}+B^{\prime}\right)$, so $A^{\prime}+B^{\prime}+H \sim A^{\prime}+B^{\prime}$, as desired.

To show that $A^{\prime}+H \sim A^{\prime}$, suppose otherwise to get a contradiction. Then $m\left(A^{\prime}+H\right)>m\left(A^{\prime}\right)$, so $m\left(\left(A^{\prime}+H\right)+B^{\prime}\right)=m\left(A^{\prime}+B^{\prime}\right)<m\left(A^{\prime}+H\right)+m\left(B^{\prime}\right)$, and 
Proposition 1.1 implies $m\left(H\left(A^{\prime}+H+B^{\prime}\right)\right)>0$. Since $A^{\prime}+H+B^{\prime} \sim A+B$, we then have $m(H(A+B))>0$, contradicting the hypothesis. Similarly $B^{\prime}+H \sim B^{\prime}$.

Lemma 5.13. If Theorem 1.3 holds under the additional assumption that $A+B$ is measurable and $H(A+B)=\{0\}$, then Theorem 1.3 holds in general.

Proof. By Lemma 5.10 we may assume $A+B$ is measurable. As in the proof of Lemma 5.10, we may assume that $(A, B)$ is irreducible and nonextendible. Write $K:=H(A+B)$. We first dispense with the case $m(K)>0$. In that case, Lemma 5.8 implies $(A, B)$ satisfies conclusion $(\mathrm{P})$ or $(\mathrm{QP})$ of Theorem 1.3 . Now assume $m(K)=0$. Let $A^{\prime} \subseteq A$ and $B^{\prime} \subseteq B$ be as in the conclusion of Lemma 5.12, so that $A^{\prime}+K \sim A^{\prime}, B^{\prime}+K \sim B^{\prime}$, and $A^{\prime}+B^{\prime}+K \sim A^{\prime}+B^{\prime} \sim A+B$. Observe that $G / K$ is infinite, since $m(K)=0$. For the remainder of the proof, write $G^{\prime}$ for $G / K$, and write $m^{\prime}$ for $m_{G^{\prime}}$.

Claim. Viewing $A^{\prime}+B^{\prime}+K$ as a subset of $G^{\prime}$, the pair $\left(A^{\prime}+K, B^{\prime}+K\right)$ is an irreducible, nonextendible, sur-critical pair for $G^{\prime}$, and $H\left(A^{\prime}+B^{\prime}+K\right)=\left\{0_{G^{\prime}}\right\}$. In particular, $m^{\prime}\left(H\left(A^{\prime}+B^{\prime}+K\right)\right)=0$, since $G^{\prime}$ is infinite.

Proof of the Claim. We first show $H\left(A^{\prime}+B^{\prime}+K\right)=\left\{0_{G^{\prime}}\right\}$ by contradiction. Supposing otherwise, there exists $z \neq 0_{G^{\prime}} \in G^{\prime}$ such that

$$
m^{\prime}\left(\left(A^{\prime}+B^{\prime}+K\right) \triangle\left(A^{\prime}+B^{\prime}+K+z\right)\right)=0 .
$$

If $t \in G$ is such that $t+K=z$, then $t \notin K$, and by Proposition 2.1 and the choice of $A^{\prime}$ and $B^{\prime}$,

$$
\begin{aligned}
m_{G}((A+B) \triangle(A+B+t)) & =m_{G}\left(\left(A^{\prime}+B^{\prime}+K\right) \triangle\left(A^{\prime}+B^{\prime}+K+t\right)\right) \\
& =m^{\prime}\left(\left(A^{\prime}+B^{\prime}+K\right) \triangle\left(A^{\prime}+B^{\prime}+K+z\right)\right) \\
& =0
\end{aligned}
$$

contradicting the definition of $K$.

If $\left(A^{\prime}+K, B^{\prime}+K\right)$ is extendible, then $m^{\prime}\left(H\left(A^{\prime}+B^{\prime}+K\right)\right)>0$ by Proposition 1.1. contradicting the previous paragraph.

If $\left(A^{\prime}+K, B^{\prime}+K\right)$ is reducible, there exist $A^{\prime \prime} \subseteq A^{\prime}+K$ and $B^{\prime \prime} \subseteq B^{\prime}+K$ such that $m^{\prime}\left(A^{\prime \prime}+K\right)=m^{\prime}\left(A^{\prime}+K\right), m^{\prime}\left(B^{\prime \prime}+K\right)=m^{\prime}\left(B^{\prime}+K\right)$, and

$$
m^{\prime}\left(A^{\prime \prime}+K+B^{\prime \prime}+K\right)<m^{\prime}\left(A^{\prime}+K+B^{\prime}+K\right) .
$$

Setting $C=A \cap\left(A^{\prime \prime}+K\right), D=B \cap\left(B^{\prime \prime}+K\right)$, we apply Proposition 2.1 and find that $m(C)=m(A), m(D)=m(B)$, and $m(C+D)<m(A)+m(B)$. This implies $(A, B)$ is reducible, contradicting our assumptions.

Now we must show that if the pair $\left(A^{\prime}+K, B^{\prime}+K\right)$ satisfies any of the conclusions $(\mathrm{P}),(\mathrm{E}),(\mathrm{K})$, or $(\mathrm{QP})$ of Theorem 1.3 , then so does the pair $(A, B)$.

If $\left(A^{\prime}+K, B^{\prime}+K\right)$ satisfies $(\mathrm{P})$ or $(\mathrm{E})$, then $H\left(\left(A^{\prime}+K\right)+\left(B^{\prime}+K\right)\right) \neq\left\{0_{G^{\prime}}\right\}$, contradicting the claim above. This case is vacuous.

If $\left(A^{\prime}+K, B^{\prime}+K\right)$ satisfies $(\mathrm{K})$, let $\chi^{\prime}: G^{\prime} \rightarrow \mathbb{T}$ be the homomorphism of conclusion $(\mathrm{K})$, and let $I, J \subseteq \mathbb{T}$ be the corresponding intervals. If $\chi=\chi^{\prime} \circ \phi$, where $\phi: G \rightarrow G^{\prime}$ is the quotient map, it is easy to check $(2.1 .1)$ that $(A, B)$ satisfies $m(A)=m\left(\chi^{-1}(I)\right), m(B)=m\left(\chi^{-1}(J)\right)$, while $A \subseteq a+\chi^{-1}(I), B \subseteq b+\chi^{-1}(J)$ for some $a, b \in G$. We then see that $(A, B)$ satisfies $(\mathrm{K})$.

If $\left(A^{\prime}+K, B^{\prime}+K\right)$ satisfies (QP) but not $(\mathrm{P})$ or $(\mathrm{E})$, let $A^{\prime}+K=A_{1}^{\prime} \cup A_{0}^{\prime}$ and $B^{\prime}+K=B_{1}^{\prime} \cup B_{0}^{\prime}$ be decompositions of $A^{\prime}+K$ and $B^{\prime}+K$ satisfying (QP) with 
quasi-period $W$, such that $A_{0}^{\prime}+B_{0}^{\prime}$ is a unique expression element of $A^{\prime}+B^{\prime}+W+K$ in $G^{\prime} / W$, and $m^{\prime}\left(A_{0}^{\prime}+K+B_{0}^{\prime}+K\right)=m^{\prime}\left(A_{0}^{\prime}+K\right)+m^{\prime}\left(B_{0}^{\prime}+K\right)$. Regarding $B_{1}^{\prime}$, $B_{0}^{\prime}, A_{1}^{\prime}$, and $A_{0}^{\prime}$ as subsets of $G$, we see that $A_{1}^{\prime} \cup A_{0}^{\prime}$ and $B_{1}^{\prime} \cup B_{0}^{\prime}$ are decompositions of $A^{\prime}+K$ and $B^{\prime}+K$ satisfying (QP) with quasi-period $W+K$.

If $m\left(A_{0}^{\prime}+K\right)=0$ or $m\left(B_{0}^{\prime}+K\right)=0$, irreducibility of $\left(A^{\prime}+K, B^{\prime}+K\right)$ implies $m\left(H\left(A^{\prime}+B^{\prime}+K\right)\right)>0$, so that $m(H(A+B))>0$, contradicting our present assumptions. Thus we may assume $m\left(A_{0}^{\prime}+K\right)>0$ and $m\left(B_{0}^{\prime}+K\right)>0$, so that Lemma 5.9 applies to $\left(A^{\prime}, B^{\prime}\right)$ and $(A, B)$. We conclude $(A, B)$ satisfies (QP).

5.5. Quasi-periodicity and complements. Throughout this subsection we make the standing assumption that $A+B$ is measurable in addition to our previous assumption that $G$ is compact.

Lemma 5.14. If $(A, B)$ is an irreducible nonextendible sur-critical pair satisfying $m(A)>0, m(B)>0$, and $m(H(A+B))=0$, then

$$
\begin{aligned}
& -B+(A+B)^{c} \sim A^{c}, \\
& -A+(A+B)^{c} \sim B^{c},
\end{aligned}
$$

and both $\left(-B,(A+B)^{c}\right)$ and $\left(-A,(A+B)^{c}\right)$ are irreducible nonextendible surcritical pairs with $m\left(H\left(-B+(A+B)^{c}\right)\right)=0$ and $m\left(H\left(-A+(A+B)^{c}\right)\right)=0$.

Note that (5.26) and (5.27) imply $-B+(A+B)^{c}$ and $-A+(A+B)^{c}$ are measurable; the proof is complicated somewhat by the hypothetical possibility that these sets are not measurable.

Lemma 5.14] is an analogue of Lemma 2.4 of [4. As shown in 4], the containment $-B+(A+B)^{c} \subseteq A^{c}$ holds unconditionally.

Proof. We first establish (5.26). Write $D$ for $-B+(A+B)^{c}$. From the unconditional containment $D \subseteq A^{c}$, we have $m_{*}(D) \leq m\left(A^{c}\right)$. If $m_{*}(D)=m\left(A^{c}\right)$, then (5.26) holds, so we assume

$$
m_{*}(D)<m\left(A^{c}\right)
$$

and derive a contradiction. Now

$$
\begin{aligned}
m(-B)+m\left((A+B)^{c}\right) & =m(B)+1-m(A+B) \\
& =m(B)+1-(m(A)+m(B)) \\
& =m\left(A^{c}\right),
\end{aligned}
$$

so (5.28) means $m_{*}\left(-B+(A+B)^{c}\right)<m(-B)+m\left((A+B)^{c}\right)$, and Proposition 1.1 implies $-B+(A+B)^{c}$ is measurable.

Let $E$ be the set of $y \in A^{c}$ such that $(y+B) \cap(A+B)^{c} \neq \varnothing$, so that $E=A^{c} \cap D$. Let $S=A^{c} \backslash E$, so that $(S+B) \cap(A+B)^{c}=\varnothing$. Nonextendibility of $(A, B)$ implies $m(S \backslash A)=0$. Since $S \subseteq A^{c}$, we have $m(S)=0$, which implies $m(E)=m\left(A^{c}\right)$. From the definition of $E$, we have $E \subseteq-B+(A+B)^{c}$. Combining this with the unconditional containment $-B+(A+B)^{c} \subseteq A^{c}$, we obtain (5.26), which is actually the desired contradiction.

We have proved (5.26), and (5.27) follows by symmetry. We finish the proof of the lemma in the following sequence of claims.

1. $m\left(H\left(-A+(A+B)^{c}\right)\right)=0$. Suppose otherwise to get a contradiction. Then $m\left(H\left(B^{c}\right)\right)>0$ by (5.27), and consequently $m(H(B))>0$. Now irreducibility of $(A, B)$ implies $m(H(A+B))>0$, contradicting our assumptions on $(A, B)$. 
2. $\left(-A,(A+B)^{c}\right)$ is a sur-critical pair. By a computation similar to (5.29), we get $m(-A)+m\left((A+B)^{c}\right)=m\left(B^{c}\right)$, so $\left(-A,(A+B)^{c}\right)$ is a sur-critical pair by (5.27).

3. $\left(-A,(A+B)^{c}\right)$ is irreducible. Assume otherwise. By Corollary 5.7, reducibility of $\left(-A,(A+B)^{c}\right)$ yields decompositions $A=A_{1} \cup A_{0}$ and $(A+B)^{c}=C_{1} \cup C_{0}$ satisfying (QP), and one of $m\left(A_{0}\right)=0$ or $m\left(C_{0}\right)=0$. If $m\left(A_{0}\right)=0$, the irreducibility of $(A, B)$ implies $m(H(A+B))>0$, contradicting the hypothesis. If $m\left(C_{0}\right)=0$, then $m\left(H\left((A+B)^{c}\right)\right)>0$, and therefore $m(H(A+B))>0$, contradicting the hypothesis.

4. $\left(-A,(A+B)^{c}\right)$ is nonextendible. Assume otherwise. Then Proposition 1.1 implies $m\left(H\left(-A+(A+B)^{c}\right)\right)>0$, contradicting the previous claim to the contrary.

We have shown that $\left(-A,(A+B)^{c}\right)$ is an irreducible nonextendible sur-critical pair satisfying $m\left(H\left(-A+(A+B)^{c}\right)\right)=0$. Reversing the roles of $A$ and $B$ yields the corresponding description of $\left(-B,(A+B)^{c}\right)$.

Lemma 5.15 (cf. 4, Lemma 5.1). Let $(A, B)$ be a sur-critical pair with $m(A)>0$ and $m(B)>0$, and let $K$ be the subgroup of $G$ generated by $A$ (so $K$ is compact and open). If $A+B$ is aperiodic, then $B$ is quasi-periodic with respect to $K$, or $B$ is contained in a coset of $K$.

Proof. Let $B=B_{1} \cup \cdots \cup B_{l}$ be a $K$-coset decomposition of $B$. Since $A \subseteq K, A+B$ is the disjoint union $\bigcup_{i=1}^{l} A+B_{i}$. Then

$$
m(A+B)=\sum_{i=1}^{l} m\left(A+B_{i}\right),
$$

while

$$
m(A+B)=m(A)+m(B)=m(A)+\sum_{i=1}^{l} m\left(B_{i}\right) .
$$

Equating the right-hand sides of (5.30) and (5.31) we get

$$
\sum_{i=1}^{l} m\left(A+B_{i}\right)-m\left(B_{i}\right)=m(A)
$$

If $m\left(A+B_{i}\right)<m(A)+m\left(B_{i}\right)$ for all $i$, then the group $W:=K \cap \bigcap_{i=1}^{l} H\left(A+B_{i}\right)$ is open by Proposition 1.1 and $A+B$ is periodic with period $W$, contradicting the hypothesis. Thus there is an $i$ such that $m\left(A+B_{i}\right) \geq m(A)+m\left(B_{i}\right)$, and (5.32) implies $m\left(A+B_{j}\right)=m\left(B_{j}\right)$ for $j \neq i$. Consequently, $A \subseteq H\left(B_{j}\right)$ and $m\left(B_{j}\right)>0$ for all $j \neq i$. This implies $K \leqslant H\left(B_{j}\right)$ and $B_{j} \sim B_{j}+K$ for all $j \neq i$. We then have that $B$ is quasi-periodic with respect to $K$ if $l>1$, and $B$ is contained in a coset of $K$ if $l=1$.

Corollary 5.16. With the hypotheses of Theorem 1.3, if $m(H(A+B))=0$ and $A$ is contained in a coset of a compact open subgroup $K_{0} \leqslant G$, then $B$ has a quasiperiodic decomposition with respect to a compact open subgroup $K \leqslant K_{0}$, or $B$ is contained in a coset of $K_{0}$.

Proof. By translation we may assume $A \subseteq K_{0}$. The hypothesis $m(H(A+B))=0$ implies $A+B$ is aperiodic. Now apply Lemma 5.15 and let $K$ be the subgroup generated by $A$. 
In the next lemma, we assume that $A=A_{1} \cup A_{0}$ has a quasi-periodic decomposition with respect to the maximal period $K$ of $A_{1}$. This is no less general than assuming that $A$ has a quasi-periodic decomposition $A=A_{1}^{\prime} \cup A_{0}^{\prime}$ with respect to some compact open subgroup $L$, since the maximal period $K$ of $A_{1}^{\prime}$ must contain $L$, and then $A_{0}^{\prime}$ is contained in a coset of $K$, since $A_{0}^{\prime}$ is contained in a coset of $L$.

Lemma 5.17 (cf. [4, Lemma 5.3). Let $(A, B)$ be an irreducible nonextendible surcritical pair with $m(A)>0, m(B)>0$, and $m(H(A+B))=0$. Let $A=A_{1} \cup A_{0}$ be a quasi-periodic decomposition with $A_{1}$ periodic with maximal period $K$. Then $B=B_{1} \cup B_{0}$, where $B_{1} \sim B_{1}+K, B_{0}$ is contained in a coset of $K$, and

(i) $A_{0}+B_{0}+K$ is a unique expression element of $A+B+K$ in $G / K$,

(ii) $m\left(A_{0}+B_{0}\right)=m\left(A_{0}\right)+m\left(B_{0}\right)$,

so $(A, B)$ satisfies conclusion (QP) of Theorem 1.3 .

Proof. Observe that

$$
m\left(A_{1}+B\right)<m(A+B)
$$

since $m\left(H\left(A_{1}+B\right)\right) \geq m(K)>0$, while $m(H(A+B))=0$. Inequality (5.33) implies

$$
\begin{aligned}
m\left(A_{1}+B\right) & \leq m(A+B)-m\left(A_{0}\right) \\
& =m(A)+m(B)-m\left(A_{0}\right) \\
& =m\left(A_{1}\right)+m(B),
\end{aligned}
$$

where the first line results from $A_{1}+B \sim A_{1}+B+K$, while $A_{0}$ is contained in a coset of $K$, the second line is due to $m(A+B)=m(A)+m(B)$, and the third results from $A=A_{1} \cup A_{0}$ being a partition of $A$. We also have

$$
0<m\left(A_{0}\right)<m(K),
$$

where $0<m\left(A_{0}\right)$ is a consequence of irreducibility of $(A, B)$ and (5.33), while $m\left(A_{0}\right)<m(K)$ follows from the hypothesis $m(H(A+B))=0$.

Claim 1. Strict inequality holds in (5.34) and $H\left(A_{1}+B\right)=K$.

Proof of Claim 1. Assume $m\left(A_{1}+B\right)=m(A+B)-m\left(A_{0}\right)$ to get a contradiction. Then $m(A+B)=m\left(A_{1}+B\right)+m\left(A_{0}\right)$, so

$$
A+B \sim\left(A_{1}+B\right) \cup\left(A_{0}+b_{0}\right)
$$

for some $b_{0} \in B$. Then (5.35) and the similarities $A_{1} \sim A_{1}+K, A_{1}+B \sim A_{1}+B+K$ imply that

$$
C:=\left\{b \in B:(A+b) \backslash\left(A_{1}+B\right) \neq \varnothing\right\}
$$

is contained in a coset of $H\left(A_{0}\right)$. If $m\left(H\left(A_{0}\right)\right)=0$, then $m(C)=0$ and $(A, B)$ is reducible, contrary to the hypothesis. If $m\left(H\left(A_{0}\right)\right)>0$, (5.36) implies $K \cap H\left(A_{0}\right) \subseteq$ $H(A+B)$, so $m(H(A+B))>0$, again contradicting our hypothesis.

Strict inequality in (5.34) implies $m\left(A_{1}+B\right)<m\left(A_{1}\right)+m(B)$. We now show $H\left(A_{1}+B\right)=K$. Write $H$ for $H\left(A_{1}+B\right)$. Applying Proposition 1.1 to $\left(A_{1}, B\right)$, we have $A_{1}+B=A_{1}+B+H$. Then $A_{1}+B+H \subseteq A+B$, so the nonextendibility of $(A, B)$ implies

$$
A_{1}+H \subset_{m} A
$$


To see that $H=K$, first note that $A_{1} \sim A_{1}+K$, so $A_{1}+B+K \sim A_{1}+B$, and we find $K \leqslant H$ by the definition of $H$. Now $A_{1}+H$ is disjoint from $A_{0}$, since otherwise $A_{1}+H=A+H$, and then $A \sim A+H$, contradicting $m(H(A+B))=0$. Thus (5.37) implies $A \backslash A_{0} \sim A_{1}+H$. The maximality of $K$ then implies $H \leqslant K$.

Let $D:=\left\{b \in B: A_{0}+b \subseteq\left(A_{1}+B\right)^{c}\right\}$, and let $D=\bigcup_{i=1}^{l} D_{i}$ be a $K$-coset decomposition of $D$.

Claim 2. $m\left(A_{0}+D_{i}\right) \geq m\left(A_{0}\right)+m\left(D_{i}\right)$ for some $i$.

Proof of Claim 2. We have $A+B=\left(A_{1}+B\right) \cup \bigcup_{i=1}^{l} A_{0}+D_{i}$. Assuming Claim 2 fails, Proposition 1.1 implies $W:=\bigcap_{i=1}^{l} H\left(A_{0}+D_{i}\right)$ is open. Then the containment $(K \cap W) \leqslant H(A+B)$ contradicts $m(H(A+B))=0$.

Let $B_{0}$ be one of the $D_{i}$ satisfying $m\left(A_{0}+D_{i}\right) \geq m\left(A_{0}\right)+m\left(D_{i}\right)$, and let $B_{1}=B \backslash B_{0}$. We aim to show that $B_{1} \sim B_{1}+K$. Using Claim 1, we apply Lemma 5.1 to $\left(A_{1}, B\right)$ and find

$$
m\left(B_{0}\right)+m\left(A_{1}+B\right) \geq m\left(A_{1}\right)+m(B) .
$$

Then

$$
\begin{array}{rlrl}
m(A+B) & \geq m\left(A_{0}+B_{0}\right)+m\left(A_{1}+B\right) & & \\
& \geq m\left(A_{0}\right)+m\left(B_{0}\right)+m\left(A_{1}+B\right) & & (\text { by Claim 2) } \\
& \geq m\left(A_{0}\right)+m\left(A_{1}\right)+m(B) & & \text { (by (5.38) }) \\
& =m(A)+m(B) . &
\end{array}
$$

Since $m(A+B)=m(A)+m(B)$, the inequalities in (5.39) are actually equalities, and

$$
A+B \sim\left(A_{0}+B_{0}\right) \cup\left(A_{1}+B\right),
$$

which is a disjoint union. The hypothesis $m(H(A+B))=0$ and (5.40) imply $A_{0}+B_{0}+K$ is a unique expression element of $A+B+K$ in $G / K$. Now (5.40) implies $B_{1}+A \subseteq A_{1}+B+K \sim A_{1}+B$. The nonextendibility of $(A, B)$ then implies $B_{1}+K \sim B_{1}$. This concludes the proof of (i). Part (ii) of the lemma follows from the first and second lines of (5.39), as the inequalities are not strict.

Lemma 5.18. Let $(A, B)$ be an irreducible nonextendible sur-critical pair for $G$ such that $m(A)>0, m(B)>0$, and $m(H(A+B))=0$. If $A+B \sim D$, where $D$ is quasi-periodic with respect to a compact open subgroup $K \leqslant G$, then $(A, B)$ satisfies conclusion (QP) of Theorem 1.3.

Proof. Write $C$ for $(A+B)^{c}$. The assumption that $A+B \sim D$, where $D$ is quasiperiodic with quasi-period $K$, implies $C \sim C^{\prime}$, where $C^{\prime} \subseteq C$ is quasi-periodic with respect to $K$, or $C^{\prime}$ is contained in a coset of $K$. Lemma 5.14 implies $(-B, C)$ is an irreducible nonextendible sur-critical pair with $m(H(-B+C))=0$, so $\left(-B, C^{\prime}\right)$ is such a pair with $m\left(H\left(-B+C^{\prime}\right)\right)=0$. If $C^{\prime}$ is contained in a coset of $K$, then Corollary 5.16 implies $-B$, and therefore $B$, is quasi-periodic with respect to $K$ or is contained in a coset of $K$. If $B$ is quasi-periodic with respect to $K$, then Lemma 5.17 implies $(A, B)$ satisfies (QP). If $B$ is contained in a coset of $K$, then Corollary 5.16 implies $A$ is contained in a coset of $K$ or $A$ is quasi-periodic with respect to $K$. Since $A+B \sim D$, which is quasi-periodic with respect to $K$, the sets $A$ and $B$ 
cannot both be contained in a coset of $K$, so $A$ is quasi-periodic with respect to $K$. Again we apply Lemma 5.17 and find $(A, B)$ satisfies (QP).

If $C^{\prime}$ is not contained in a coset of $K$, then $C^{\prime}$ is quasi-periodic with respect to $K$, and Lemma 5.17 implies $\left(-B, C^{\prime}\right)$ satisfies conclusion (QP) of Theorem 1.3 . Then $-B$ is contained in a coset of $K$ or $-B$ is quasi-periodic with respect to $K$, and as above we conclude that $(A, B)$ satisfies (QP).

5.6. The $e$-transform. For $A, B \subseteq G$ and $e \in G$, form the pair $\left(A_{e}, B_{e}\right)$, where

$$
A_{e}:=A \cup(B+e), B_{e}:=(A-e) \cap B .
$$

This is the Dyson $e$-transform, whose properties are well documented. In particular,

$$
\begin{aligned}
A_{e}+B_{e} & \subseteq A+B, \\
m\left(A_{e}\right)+m\left(B_{e}\right) & =m(A)+m(B),
\end{aligned}
$$

whenever $B_{e}$ is nonempty. See [12], [17], or [21] for further exposition.

The proof of Proposition 1.2 in $\left[12\right.$ relies on a sequence of pairs $\left(A^{(n)}, B^{(n)}\right)$ successively derived by $e$-transform, where $\lim _{n \rightarrow \infty} m\left(B^{(n)}\right)=0$ and $m\left(B^{(n)}\right)>0$ for all $n$. The next lemma facilitates the same construction for some pairs $(A, B)$ where the ambient group is disconnected.

Lemma 5.19. If $A, B \subseteq G$ have positive Haar measure, $A+B$ is measurable, $m(A)+m(B) \leq 1$, and $A+B$ is aperiodic, then there is an $e \in G$ such that $B_{e}:=(A-e) \cap B \neq \varnothing$ and $m\left(B_{e}\right) \leq(1-m(B)) m(B)$.

In particular, the conclusion holds when $m(H(A+B))=0$. The hypothesis " $A+B$ is aperiodic" cannot be omitted; the conclusion fails when $B$ is a coset of a compact open subgroup $K \leqslant G$ and $A$ is a union of cosets of $K$.

Proof. Write $f(z)=m((A-z) \cap B)$, so that $f: G \rightarrow[0,1]$ is a continuous function and $\int f d m=m(A) m(B)([12$, Lemma 1). Note that $(A-z) \cap B$ is nonempty exactly when $z \in A-B$. Consider $S:=\{z: f(z)>0\}$, which is contained in $A-B$. If $S \neq A-B$, then there is an $e \in A-B$ with $f(e)=0$, meaning $B_{e} \neq \varnothing$ and $m\left(B_{e}\right)=0$, so we are done. If $S=A-B$, consider the average

$$
\frac{1}{m(S)} \int_{S} f d m=\frac{1}{m(A-B)} m(A) m(B) .
$$

We estimate $m(A-B)$. If $m(A-B)<m(A)+m(B)$, then Corollary 5.4 implies $A+B$ is periodic, contradicting the hypotheses. Now $m(A-B) \geq m(A)+m(B)$, and equation (5.43) implies

$$
\begin{aligned}
\frac{1}{m(S)} \int_{S} f d m & \leq \frac{m(A)}{m(A)+m(B)} m(B) \\
& =\left(1-\frac{m(B)}{m(A)+m(B)}\right) m(B) \\
& \leq(1-m(B)) m(B),
\end{aligned}
$$

where we used $m(A)+m(B) \leq 1$ in the last line. So there is an $e \in G$ such that $0<f(e)<(1-m(B)) m(B)$. Now $0<m\left(B_{e}\right) \leq(1-m(B)) m(B)$ by the definition of $f$. 
5.7. The key lemma. The proof of the next lemma follows from part of the argument of [4], $\S 6$, Subcase 1.

Lemma 5.20. Suppose $(A, B)$ is an irreducible, nonextendible, sur-critical pair having $m(A)>0, m(B)>0, m(H(A+B))=0$, and there exists $e \in G$ such that $m_{*}\left(A_{e}+B_{e}\right)<m(A)+m(B)$. Then $(A, B)$ satisfies conclusion (QP) of Theorem 1.3 with $K=H\left(A_{e}+B_{e}\right)$.

Proof. Without loss of generality assume $e=01$ so $A_{e}=A \cup B$, and $B_{e}=A \cap B$. Since $m\left(A_{e}\right)+m\left(B_{e}\right)=m(A)+m(B)$, we apply Proposition 1.1 to $\left(A_{e}, B_{e}\right)$ and find

$$
A_{e}+B_{e}=A_{e}+B_{e}+H
$$

while $H=H\left(A_{e}+B_{e}\right)$ is compact and open.

Claim 1. $(A \cap B)+H \sim A \cap B$.

Proof of Claim 1. If $b \in A \cap B$, then $A+b \subseteq A_{e}+B_{e}=A_{e}+B_{e}+H$, so $A+b+H \subseteq$ $A+B$. Nonextendibility of $(A, B)$ then implies $b+H \subset_{m} B$. Likewise, if $a \in A \cap B$, then $a+B \subseteq A_{e}+B_{e}$, so $a+H \subset_{m} A$. The claim follows.

Partition $A$ as $A=A_{0} \cup A_{1} \cup A_{2}$, where

$$
\begin{aligned}
& A_{0}:=(A \cap(B+H)) \backslash[(A \cap B)+H], \\
& A_{1}:=A \cap[(A \cap B)+H], \\
& A_{2}:=A \backslash\left(A_{0} \cup A_{1}\right) .
\end{aligned}
$$

Partition $B$ similarly, as $B_{0}:=(B \cap(A+H)) \backslash(A \cap B), B_{1}:=B \cap[(A \cap B)+H]$, and $B_{2}:=B \backslash\left(B_{0} \cup B_{1}\right)$.

Claim 2. $A_{0}+H=B_{0}+H$.

Proof of Claim 2. The definitions of $A_{0}$ and $B_{0}$ imply $A+H$ contains $B_{0}$, while $A_{1}+H$ and $A_{2}+H$ are disjoint from $B_{0}$. Thus $B_{0} \subseteq A_{0}+H$. Similarly, $A_{0} \subseteq$ $B_{0}+H$.

We now consider three cases.

Case 1. There exists $b \in B_{2}$ such that $(A+b) \nsubseteq\left(A_{e}+B_{e}\right)$. In this case (5.44) implies that there is a $b \in B_{2}$ and an $a_{0} \in A$ such that $a_{0}+((b+H) \cap B)$ is disjoint from $A_{e}+B_{e}$. Fix such $b$, and set $B_{0}=(b+H) \cap B$. Note that $B_{0}=A_{e} \cap(b+H)$, by the definition of $B_{2}$. Then Lemma [5.1, applied to $\left(A_{e}, B_{e}\right)$, implies

$$
m\left(A_{e}+B_{e}\right)+m\left(B_{0}\right) \geq m\left(A_{e}\right)+m\left(B_{e}\right)=m(A+B) .
$$

The containment $A_{e}+B_{e} \subseteq A+B$, (5.45), and the definition of $B_{0}$ imply

$$
A+B \sim\left(A_{e}+B_{e}\right) \cup\left(a_{0}+B_{0}\right) .
$$

Since $A_{e}+B_{e}$ is $H$-periodic, the set $\left(A_{e}+B_{e}\right) \cup\left(a_{0}+B_{0}\right)$ is quasi-periodic. By (5.46) and Lemma [5.18, $(A, B)$ satisfies conclusion (QP) of Theorem 1.3 with $K=H$. We are done with Case 1.

Case 2. There exists $a \in A_{2}$ such that $(a+B) \nsubseteq\left(A_{e}+B_{e}\right)$. We argue as in Case 1 , reversing the roles of $A$ and $B$.

\footnotetext{
${ }^{1}$ Write $C=A-e, D=B$, so applying the $e$-transform to $(C, D)$ with $e=0$ yields $\left(C^{\prime}, D^{\prime}\right)=$ $((A-e) \cup B,(A-e) \cap B)=\left(A_{e}-e, B_{e}\right)$.
} 


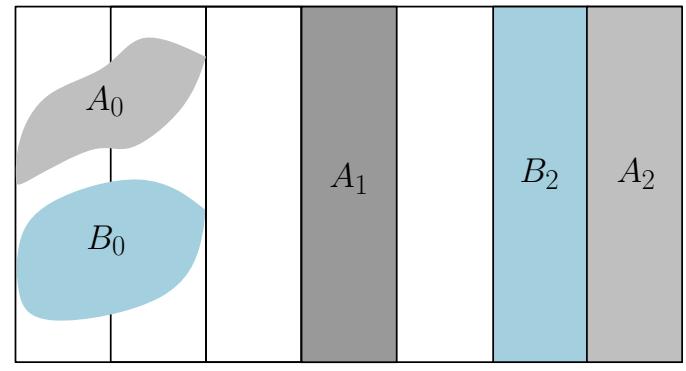

Figure 1

Case 3. For all $a \in A_{2}$ and $b \in B_{2}, A+b \subseteq A_{e}+B_{e}$ and $a+B \subseteq A_{e}+B_{e}$. In this case, nonextendibility of $(A, B)$ and the containment $A_{e}+B_{e}+H \subseteq A+B$ imply $b+H \subset_{m} B$ for all $b \in B_{2}$, and $a+H \subset_{m} A$ for all $a \in A_{2}$.

By Claims 1 and 2 and the definitions of the $A_{i}$, we now have $A=A_{0} \cup A_{1} \cup A_{2}$, where $A_{1}+H \sim A_{1}, A_{2}+H \sim A_{2}$, and $A_{0} \cap B=\varnothing$. Similarly, $B=B_{0} \cup B_{1} \cup B_{2}$, where $B_{1}+H=B_{1}, B_{2}+H=B_{2}$, and $B_{0} \cap A=\varnothing$. Also, $A_{0}+H=B_{0}+H$. Figure 1 depicts this scenario: the vertical rectangles are cosets of $H$, while contrary to the conclusion of the lemma, $A_{0}$ and $B_{0}$ are shown as occupying two cosets of $H$. Our immediate goal is to show that $A+B$ has a quasi-periodic decomposition with quasi-period $H$. Let

$$
A_{0}=\bigcup_{i=1}^{l} A_{0, i}, B_{0}=\bigcup_{i=1}^{l} B_{0, i}
$$

be $H$-coset decompositions of $A_{0}$ and $B_{0}$, with $A_{0, i}+H=B_{0, i}+H=: H_{i}$. Then $A_{e} \cap H_{i}=\left(A_{0, i} \cup B_{0, i}\right)$, which is a disjoint union, for each $i$. Lemma 5.1, applied to $\left(A_{e}, B_{e}\right)$, implies

$$
m\left(A_{0, i}\right)+m\left(B_{0, i}\right)+m\left(A_{e}+B_{e}\right) \geq m\left(A_{e}\right)+m\left(B_{e}\right)=m(A+B),
$$

for all $i$.

Claim 3. There is a pair $(i, j)$ such that $H_{i}+H_{j}$ is disjoint from $A_{e}+B_{e}$, and $m\left(A_{0, j}+B_{0, i}\right) \geq m\left(A_{0, j}\right)+m\left(B_{0, i}\right)$ or $m\left(A_{0, i}+B_{0, j}\right) \geq m\left(A_{0, i}\right)+m\left(B_{0, j}\right)$.

Proof of Claim 3. Assuming otherwise, Proposition 1.1 implies the group $W:=$ $\bigcap_{(i, j)} H\left(A_{0, j}+B_{0, i}\right) \cap H\left(A_{0, i}+B_{0, j}\right)$ is open, where the intersection is over all pairs $(i, j)$ such that $H_{i}+H_{j}$ is disjoint from $A_{e}+B_{e}$. Then the containment $(W \cap H) \leqslant H(A+B)$ contradicts $m(H(A+B))=0$.

Assume, to get a contradiction, that $A+B$ is not quasi-periodic with quasiperiod $H$. Let $(i, j)$ be a pair satisfying the conclusion of Claim 3 , and let $\left(i^{\prime}, j^{\prime}\right)$ be another pair such that the sets

$$
\left(A_{0, i}+B_{0, j}\right) \cup\left(A_{0, j}+B_{0, i}\right) \text { and }\left(A_{0, i^{\prime}}+B_{0, j^{\prime}}\right) \cup\left(A_{0, j^{\prime}}+B_{0, i^{\prime}}\right)
$$

occupy distinct cosets of $H$ and are disjoint from $A_{e}+B_{e}$. Writing

$$
\begin{aligned}
M & :=\max \left\{m\left(A_{0, i}\right), m\left(A_{0, j}\right), m\left(B_{0, i}\right), m\left(B_{0, j}\right)\right\}, \\
M^{\prime} & :=\max \left\{m\left(A_{0, i^{\prime}}\right), m\left(A_{0, j^{\prime}}\right), m\left(B_{0, i^{\prime}}\right), m\left(B_{0, j^{\prime}}\right)\right\},
\end{aligned}
$$


and $S$ for $\left\{i, i^{\prime}, j, j^{\prime}\right\}$, we estimate $m(A+B)$ from below by

$$
m(A+B) \geq M+M^{\prime}+m\left(A_{e}+B_{e}\right) .
$$

Combining (5.48) with (5.47) yields

$$
M+M^{\prime} \leq m\left(A_{0, s}\right)+m\left(B_{0, s}\right) \text { for all } s \in S,
$$

so in particular, $M+M^{\prime} \leq \min _{s \in S}\left\{m\left(A_{0, s}\right)+m\left(B_{0, s}\right)\right\}$. Assume, without loss of generality, that $M \leq M^{\prime}$. Since

$$
\min _{s \in S}\left\{m\left(A_{0, s}\right)+m\left(B_{0, s}\right)\right\} \leq \min _{s \in S} \min \left\{m\left(A_{0, s}\right), m\left(B_{0, s}\right)\right\}+M^{\prime},
$$

(5.49) implies

$$
M=\min _{s \in S} \min \left\{m\left(A_{0, s}\right), m\left(B_{0, s}\right)\right\} .
$$

From the definition of $M$, (5.50) implies

$$
M=m\left(A_{0, i}\right)=m\left(A_{0, j}\right)=m\left(B_{0, i}\right)=m\left(B_{0, j}\right) .
$$

Combining (5.51) with (5.49) we conclude $M=M^{\prime}$, and

$$
m\left(A_{0, s}\right)=m\left(A_{0, t}\right)=m\left(B_{0, s}\right)=m\left(B_{0, t}\right) \text { for all } s, t \in S .
$$

Claim 3 and (5.52) imply $m\left(\left(A_{0, i}+B_{0, j}\right) \cup\left(A_{0, j}+B_{0, i}\right)\right) \geq m\left(A_{0, i}\right)+m\left(B_{0, i}\right)$. Again estimating $m(A+B)$, we have

$$
\begin{aligned}
m(A+B) \geq & m\left(A_{e}+B_{e}\right)+m\left(\left(A_{0, i}+B_{0, j}\right) \cup\left(A_{0, j}+B_{0, i}\right)\right) \\
& +m\left(\left(A_{0, i^{\prime}}+B_{0, j^{\prime}}\right) \cup\left(A_{0, j^{\prime}}+B_{0, i^{\prime}}\right)\right) \\
> & m\left(A_{e}+B_{e}\right)+m\left(A_{0, i}\right)+m\left(B_{0, i}\right) \\
\geq & m(A)+m(B),
\end{aligned}
$$

where the last line follows from (5.47). The strict inequality in (5.53) is the desired contradiction. This concludes the analysis of Case 3 and the proof of the lemma.

\subsection{Intersections and measure.}

Lemma 5.21. If $A, B \subseteq G$ have $m(A)>0$ and $m(B)>0$, there are sets $A^{\prime} \subseteq A$, $B^{\prime} \subseteq B$ with $m\left(A^{\prime}\right)=m(A)$ and $m\left(B^{\prime}\right)=m(B)$, such that $m\left(\left(A^{\prime}-t\right) \cap B^{\prime}\right)>0$ whenever $\left(A^{\prime}-t\right) \cap B^{\prime}$ is nonempty.

Proof. By Theorem A of [15], there is a sequence of neighborhoods $U_{n}$ of the identity $0 \in G$ such that for $m$-almost all $a \in A$ and $b \in B$,

$$
\lim _{n \rightarrow \infty} \frac{m\left(A \cap\left(U_{n}+a\right)\right)}{m\left(U_{n}\right)}=1 \text { and } \lim _{n \rightarrow \infty} \frac{m\left(B \cap\left(U_{n}+b\right)\right)}{m\left(U_{n}\right)}=1 .
$$

Let $A^{\prime} \subseteq A$ and $B^{\prime} \subseteq B$ be the sets of points in $A$ and $B$, respectively, satisfying (5.54), so that $m\left(A^{\prime}\right)=m(A)$ and $m\left(B^{\prime}\right)=m(B)$. If $t \in G$ and $\left(A^{\prime}-t\right) \cap B^{\prime}$ is nonempty, assume without loss of generality that $0 \in\left(A^{\prime}-t\right) \cap B^{\prime}$. Now (5.54) implies that for $n$ sufficiently large,

$$
m\left(\left(A^{\prime}-t\right) \cap U_{n}\right)>m\left(U_{n}\right) / 2 \text { and } m\left(B^{\prime} \cap U_{n}\right)>m\left(U_{n}\right) / 2,
$$

so $m\left(\left(A^{\prime}-t\right) \cap B^{\prime} \cap U_{n}\right)>0$. Thus $m\left(\left(A^{\prime}-t\right) \cap B^{\prime}\right)>0$. 
Remark. Theorem A of [15] is proved in [16]. We outline a way to find neighborhoods $U_{n}$ satisfying (5.54): by Proposition 2.42 of $[2]$ and the ensuing remarks, there is a sequence of neighborhoods $V_{n}$ of the identity $0 \in G$ such that the functions

$$
f_{n}(z):=m\left(A \cap\left(V_{n}+z\right)\right) / m\left(V_{n}\right), g_{n}(z):=m\left(B \cap\left(V_{n}+z\right)\right) / m\left(V_{n}\right)
$$

converge in $L^{1}(m)$ to the functions $1_{A}$ and $1_{B}$, respectively. One may then choose a subsequence $\left\{U_{n}\right\}_{n \in \mathbb{N}}$ of $\left\{V_{n}\right\}_{n \in \mathbb{N}}$ so that the corresponding subsequences of $f_{n}$ and $g_{n}$ converge $m$-almost everywhere. These $U_{n}$ will satisfy (5.54).

\section{The MAIN ARgument}

6.1. The sequence of $e$-transforms. As in [12, we construct a sequence of pairs by successively applying the $e$-transform (\$5.6).

Lemma 6.1. Let $(A, B)$ be an irreducible, nonextendible pair satisfying the hypotheses of Theorem 1.3 such that $m(H(A+B))=0$. Then at least one of the following holds.

(i) There is a sequence of pairs $\left(A^{(n)}, B^{(n)}\right), n=0,1,2, \ldots$, such that $A^{(0)}=$ $A, B^{(0)}=B$, and for all $n \geq 1$,

(i.1) the pair $\left(A^{(n)}, B^{(n)}\right)$ is derived from $\left(A^{(n-1)}, B^{(n-1)}\right)$ by e-transform,

(i.2) $A^{(n)}+B^{(n)} \sim A+B$, and

(i.3) $0<m\left(B^{(n)}\right) \leq\left(1-m\left(B^{(n-1)}\right)\right) m\left(B^{(n-1)}\right)$.

(ii) $(A, B)$ satisfies conclusion (QP) of Theorem 1.3 ,

Proof. Suppose $\left(A^{(n)}, B^{(n)}\right)$ satisfies the description in (i) for $n=0, \ldots, k$. We will show that there is a pair $\left(A^{(k+1)}, B^{(k+1)}\right)$ satisfying (i.1)-(i.3) with $n=k+1$, or (ii) holds. Note that $\left(A^{(k)}, B^{(k)}\right)$ is not extendible, as (i.2) implies $H\left(A^{(k)}+B^{(k)}\right)=\{0\}$.

If $\left(A^{(k)}, B^{(k)}\right)$ is reducible, Corollary [5.7 says that $\left(A^{(k)}, B^{(k)}\right)$ satisfies (QP) of Theorem 1.3. Since $A+B \sim A^{(k)}+B^{(k)}$, Lemma 5.18 implies $(A, B)$ satisfies conclusion (QP) of Theorem 1.3, and we have (ii).

If $\left(A^{(k)}, B^{(k)}\right)$ is irreducible, apply Lemma 5.21 to find $C \subseteq A^{(k)}$ and $D \subseteq B^{(k)}$ such that $m(C)+m(D)=m\left(A^{(k)}\right)+m\left(B^{(k)}\right)$ and $(C-e) \cap D \neq \varnothing$ implies $m((C-e) \cap D)>0$. By Lemma 5.19, there is an $e \in G$ such that

$$
0<m\left(D_{e}\right) \leq(1-m(D)) m(D) .
$$

Take $B^{(k+1)}=\left(B^{(k)}\right)_{e}$ and $A^{(k+1)}=\left(B^{(k)}\right)_{e}$. If

$$
m\left(A^{(k+1)}+B^{(k+1)}\right)=m(A)+m(B)
$$

we have (i.1)-(i.3) with $n=k+1$, since $B^{(k+1)} \sim D_{e}$. Otherwise,

$$
m\left(A^{(k+1)}+B^{(k+1)}\right)<m\left(A^{(k+1)}\right)+m\left(B^{(k+1)}\right)
$$

and Lemma 5.20 implies $A^{(k)}+B^{(k)}(\sim A+B)$ is quasi-periodic. Now Lemma 5.18 implies $(A, B)$ satisfies conclusion (QP) of Theorem 1.3. We conclude (ii).

If the construction of $\left(A^{(n)}, B^{(n)}\right)$ yields conclusion (ii) for some $n$, we are done. Otherwise, we have (i).

We now prove a special case of Theorem 1.3 .

Proposition 6.2. Theorem 1.3 holds under the additional assumptions that $(A, B)$ is irreducible and nonextendible, $A+B$ is measurable, and $H(A+B)=\{0\}$. 
Proof. If $G$ is finite, then conclusion (P) of Theorem 1.3 holds. If $G$ is infinite, the hypotheses of Lemma 6.1 are satisfied. If (ii) holds in the conclusion of Lemma 6.1, then we are done. Now we must analyze the case where conclusion (i) holds in Lemma 6.1. Let $\left(A^{(n)}, B^{(n)}\right)$ be a sequence of pairs satisfying (i) of Lemma 6.1. We argue as in the proof of Proposition 1.2 in [12].

Claim. For every neighborhood $U$ of $0 \in G$, there exists $n$ with $B^{(n)}-B^{(n)} \subseteq U$.

Proof of the Claim. Suppose not. Then, since $B^{(n+1)} \subseteq B^{(n)}$ for all $n$, there exists a neighborhood $U$ of 0 and elements $x_{n}, y_{n} \in B^{(n)}$ such that $x_{n}-y_{n} \notin U$ for all $n \in \mathbb{N}$. Since $G$ is compact, the sequences $x_{n}, y_{n}$ have limit points $x, y$, with $x-y \neq 0$. Our choice of $x_{n}$ and $y_{n}$ implies

$$
A^{(n)} \subseteq\left(A^{(n)}+B^{(n)}-y_{n}\right) \cap\left(A^{(n)}+B^{(n)}-x_{n}\right) .
$$

Condition (i.3) in Lemma 6.1 implies $\lim _{n \rightarrow \infty} m\left(B^{(n)}\right)=0$, so (5.42) implies $\lim _{n \rightarrow \infty} m\left(A^{(n)}\right)=m(A)+m(B)$. Then

$$
\begin{aligned}
m((A+B) \cap(A+B+y & -x)) \geq \liminf _{n \rightarrow \infty} m\left((A+B) \cap\left(A+B+y_{n}-x_{n}\right)\right) \\
& =\liminf _{n \rightarrow \infty} m\left(\left(A^{(n)}+B^{(n)}-y_{n}\right) \cap\left(A^{(n)}+B^{(n)}-x_{n}\right)\right) \\
& \geq \lim _{n \rightarrow \infty} m\left(A^{(n)}\right) \\
& =m(A)+m(B) \\
& =m(A+B),
\end{aligned}
$$

where the second line uses translation-invariance of $m$ and the third uses (6.2). Now $A+B+(y-x) \sim A+B$, contradicting the assumption $H(A+B)=\{0\}$.

We now consider two separate cases. Corollary 7.9 of [8] implies these two cases cover all possible compact abelian groups $G$.

Case 1. $G$ has open subgroups of arbitrarily small measure. By the Claim, there is a compact open subgroup $K$ and $n \in \mathbb{N}$ such that $B^{(n)}-B^{(n)} \subseteq K$, while $m\left(A^{(n)}\right)>m(K)$. Then $B^{(n)}$ is contained in a coset of $K$, while $A^{(n)}$ is not. Then Corollary 5.16 implies $A^{(n)}$ is quasi-periodic, and Lemma 5.17 implies $A^{(n)}+B^{(n)}$ is quasi-periodic. Since $A+B \sim A^{(n)}+B^{(n)}$, Lemma 5.18 implies $(A, B)$ satisfies (QP) of Theorem 1.3 .

Case 2. G has an open connected subgroup. Let $G_{0}$ be an open connected subgroup of $G$. By the Claim, we can choose $n$ sufficiently large such that $B^{(n)}$ is contained in a coset of $G_{0}$. If $A^{(n)}$ is contained in a coset of $G_{0}$, then by the construction of $A^{(n)}, A$ and $B$ are each contained in a coset of $G_{0}$. Then Proposition 1.2 implies the existence of a continuous surjective homomorphism $\chi: G_{0} \rightarrow \mathbb{T}$ and intervals $I, J \subseteq \mathbb{T}$ such that $A \subseteq a+\chi^{-1}(I), B \subseteq b+\chi^{-1}(J)$, for some $a, b \in G$, such that $m(A)=m\left(\chi^{-1}(I)\right)$ and $m(B)=m\left(\chi^{-1}(J)\right)$. From this we conclude $(\mathrm{K})$ in Theorem 1.3. If $A^{(n)}$ is not contained in a coset of $G_{0}$, we argue as in Case 1 and find that $(A, B)$ satisfies $(\mathrm{QP})$.

6.2. Proof of Theorem 1.3. If $(A, B)$ is extendible, we have conclusion (E). If $(A, B)$ is nonextendible and reducible, Lemma 5.6 implies $(A, B)$ satisfies conclusion (QP). If $(A, B)$ is irreducible and nonextendible, Lemma 5.13 allows us to assume that $A+B$ is measurable and $H(A+B)=\{0\}$. In that case, Proposition 6.2 implies the conclusion of Theorem 1.3 . 


\section{When $G$ IS NOT COMPACT}

A structure theorem for locally compact abelian groups ([8], Theorem 24.30) says that such a group $G$ is isomorphic (as a topological group) to $\mathbb{R}^{n} \times L$, where $L$ is a locally compact group containing a compact open subgroup, and $n$ is a nonnegative integer. The study of the equation

$$
m_{*}(A+B)=m(A)+m(B)
$$

depends heavily on $n$. Theorem 5 of [12] says that when $n \geq 1$,

$$
m_{*}(A+B)^{1 / n} \geq m(A)^{1 / n}+m(B)^{1 / n}
$$

for measurable subsets $A$ and $B$ of $G$, and Theorem 6 of [12] classifies the pairs for which equality holds in (7.2), generalizing Theorem 2 of [7] to the case where $L$ is nontrivial. When $n>1$ and $A$ and $B$ both have positive measure, (7.2) implies $m_{*}(A+B)>m(A)+m(B)$, so there are no such pairs satisfying (7.1) when $n>1$. When $n=1$, Theorem 6 of 12 says that if $A$ and $B$ have positive measure, then (7.1) holds if and only if there are closed intervals $I, J \subseteq \mathbb{R}$, a compact open subgroup $K \leqslant L$, and $a, b \in G$ such that $A \subseteq a+(I \times K), B \subseteq b+(J \times K)$, and $m(A)=m(I \times K), m(B)=m(J \times K)$.

Our study of equation (7.1) is thus reduced to groups $G$ having a compact open subgroup. We assume $A$ and $B$ have finite measure, since otherwise (7.1) is satisfied. One can easily check that $A$ and $B$ have compact closures under these assumptions. Replacing $G$ by the group generated by $A \cup B$, we can assume that $G$ is compactly generated. A compactly generated group with a compact open subgroup is isomorphic to $\mathbb{Z}^{d} \times K$ for some nonnegative integer $d$ and some compact group $K$ ([8], Theorem 9.8). For such groups $G$, it is then routine to verify that Theorem 1.3 holds with no modification of the conclusion. This is the content of the following corollary.

Corollary 7.1. The conclusion of Theorem 1.3 holds under the weaker assumption that $G$ has a compact open subgroup and $m_{*}(A+B)=m(A)+m(B)<\infty$.

\section{ACKNOWLEDGEMENTS}

This work was conducted mostly while the author was a postdoctoral fellow in the Department of Mathematics at the University of British Columbia. The author thanks Izabella Łaba and Malabika Pramanik for financial support and helpful discussions. Discussions with Michael Björklund and Alexander Fish provided motivation for this work.

\section{REFERENCES}

[1] Yuri Bilu, The $(\alpha+2 \beta)$-inequality on a torus, J. London Math. Soc. (2) 57 (1998), no. 3, 513-528, DOI 10.1112/S0024610798006024. MR.1659821(99j:11009)

[2] Gerald B. Folland, A course in abstract harmonic analysis, Studies in Advanced Mathematics, CRC Press, Boca Raton, FL, 1995. MR1397028 (98c:43001)

[3] David J. Grynkiewicz, Quasi-periodic decompositions and the Kemperman structure theorem, European J. Combin. 26 (2005), no. 5, 559-575, DOI 10.1016/j.ejc.2004.06.011. MR2126639 (2005m:05222)

[4] David J. Grynkiewicz, A step beyond Kemperman's structure theorem, Mathematika 55 (2009), no. 1-2, 67-114, DOI 10.1112/S0025579300000966. MR2573603 (2010k:11161)

[5] Yahya Ould Hamidoune and Øystein J. Rødseth, An inverse theorem mod $p$, Acta Arith. 92 (2000), no. 3, 251-262. MR.1752029(2001c:11114) 
[6] Yahya Ould Hamidoune, Oriol Serra, and Gilles Zémor, On the critical pair theory in $\mathbb{Z} / p \mathbb{Z}$, Acta Arith. 121 (2006), no. 2, 99-115, DOI 10.4064/aa121-2-1. MR2216136(2007a:11145)

[7] R. Henstock and A. M. Macbeath, On the measure of sum-sets. I. The theorems of Brunn, Minkowski, and Lusternik, Proc. London Math. Soc. (3) 3 (1953), 182-194. MR0056669 $(15,109 \mathrm{~g})$

[8] Edwin Hewitt and Kenneth A. Ross, Abstract harmonic analysis. Vol. I, 2nd ed., Grundlehren der Mathematischen Wissenschaften [Fundamental Principles of Mathematical Sciences], vol. 115, Springer-Verlag, Berlin, 1979. Structure of topological groups, integration theory, group representations. MR551496 (81k:43001)

[9] Renling Jin, Characterizing the structure of $A+B$ when $A+B$ has small upper Banach density, J. Number Theory 130 (2010), no. 8, 1785-1800, DOI 10.1016/j.jnt.2010.02.008. MR2651155(2011k:11011)

[10] J. H. B. Kemperman, On small sumsets in an abelian group, Acta Math. 103 (1960), 63-88. MR0110747 (22 \#1615)

[11] J. H. B. Kemperman, On products of sets in a locally compact group, Fund. Math. 56 (1964), 51-68. MR0202913(34 \#2772)

[12] Martin Kneser, Summenmengen in lokalkompakten abelschen Gruppen, Math. Z. 66 (1956), 88-110 (German). MR0081438(18,403a)

[13] A. M. Macbeath, On measure of sum sets. II. The sum-theorem for the torus, Proc. Cambridge Philos. Soc. 49 (1953), 40-43. MR0056670 (15,110a)

[14] D. A. Moskvin, G. A. Frel̆man, and A. A. Judin, Inverse problems of additive number theory and local limit theorems for lattice random variables, Number-theoretic studies in the Markov spectrum and in the structural theory of set addition (Russian), Kalinin. Gos. Univ., Moscow, 1973, pp. 148-162 (Russian). MR0435005 (55 \#7967)

[15] Bruno J. Mueller, Three results for locally compact groups connected with Haar measure density theorem, Proc. Amer. Math. Soc. 16 (1965), 1414-1416. MR0200385 (34 \#280)

[16] Bruno Müller, Eine Verschärfung für Abschätzungen von Summenmengen in lokalkompakten Gruppen, Math. Z. 78 (1962), 199-204 (German). MR0150234 (27 \#235)

[17] Melvyn B. Nathanson, Additive number theory, Graduate Texts in Mathematics, vol. 165, Springer-Verlag, New York, 1996. Inverse problems and the geometry of sumsets. MR.1477155 (98f:11011)

[18] D. Raikov, On the addition of point-sets in the sense of Schnirelmann, Rec. Math. [Mat. Sbornik] N.S. 5(47) (1939), 425-440. MR0001776 (1,296b)

[19] Hans Reiter and Jan D. Stegeman, Classical harmonic analysis and locally compact groups, 2nd ed., London Mathematical Society Monographs. New Series, vol. 22, The Clarendon Press Oxford University Press, New York, 2000. MR,1802924 (2002d:43005)

[20] A. Shields, Sur la mesure d'une somme vectorielle, Fund. Math. 42 (1955), 57-60 (French). MR0072201 (17,245f)

[21] Terence Tao and Van Vu, Additive combinatorics, Cambridge Studies in Advanced Mathematics, vol. 105, Cambridge University Press, Cambridge, 2006. MR2289012 (2008a:11002)

[22] A. G. Vosper, Addendum to "The critical pairs of subsets of a group of prime order", J. London Math. Soc. 31 (1956), 280-282. MR0078368 (17,1182g)

[23] A. G. Vosper, The critical pairs of subsets of a group of prime order, J. London Math. Soc. 31 (1956), 200-205. MR.0077555 (17,1056c)

Department of Mathematics, The University of British Columbia, Room 121, 1984 Mathematics Road, Vancouver, British Columbia, Canada V6T 1 Z2

Current address: Department of Mathematics, University of Denver, John Greene Hall, Room 203, 2360 S. Gaylord Street, Denver, Colorado 80208

E-mail address: John.Griesmer@du.edu 